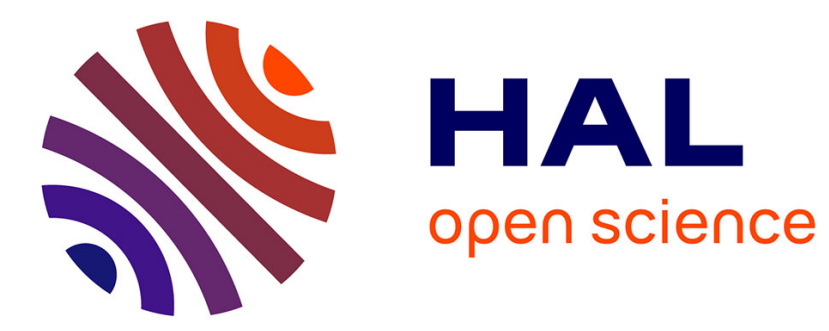

\title{
A numerical reduced model for thin liquid films sheared by a gas flow
}

\author{
Gianluca Lavalle, Jean-Paul Vila, G. Blanchard, Claire Laurent, François
}

Charru

\section{> To cite this version:}

Gianluca Lavalle, Jean-Paul Vila, G. Blanchard, Claire Laurent, François Charru. A numerical reduced model for thin liquid films sheared by a gas flow. Journal of Computational Physics, 2015, 301, pp.119140. 10.1016/j.jcp.2015.08.018 . hal-01597465

\section{HAL Id: hal-01597465 \\ https://hal.science/hal-01597465}

Submitted on 28 Sep 2017

HAL is a multi-disciplinary open access archive for the deposit and dissemination of scientific research documents, whether they are published or not. The documents may come from teaching and research institutions in France or abroad, or from public or private research centers.
L'archive ouverte pluridisciplinaire HAL, est destinée au dépôt et à la diffusion de documents scientifiques de niveau recherche, publiés ou non, émanant des établissements d'enseignement et de recherche français ou étrangers, des laboratoires publics ou privés. 


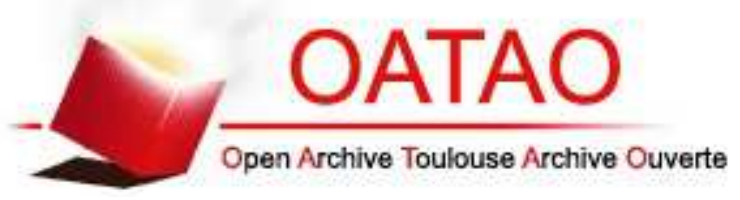

\section{Open Archive TOULOUSE Archive Ouverte (OATAO)}

OATAO is an open access repository that collects the work of Toulouse researchers and makes it freely available over the web where possible.

This is an author-deposited version published in : http://oatao.univ-toulouse.fr/ Eprints ID : 18393

To link to this article : DOI: $10.1016 / j . j c p .2015 .08 .018$

URL : http://dx.doi.org/10.1016/j.jcp.2015.08.018

To cite this version : Lavalle, Gianluca and Vila, Jean-Paul and

Blanchard, G. and Laurent, Claire and Charru, François A numerical reduced model for thin liquid films sheared by a gas flow. (2015) Journal of Computational Physics, vol. 301. pp. 119-140. ISSN 0021-9991

Any correspondence concerning this service should be sent to the repository administrator: staff-oatao@ listes-diff.inp-toulouse.fr 


\title{
A numerical reduced model for thin liquid films sheared by a gas flow
}

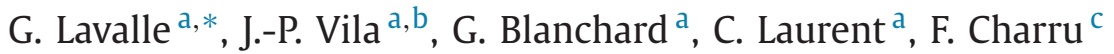 \\ a ONERA, Aerodynamics and Energetics Modeling Department, 31055 Toulouse, France \\ b Institut de Mathématiques de Toulouse, INSA, GMM, 31055 Toulouse, France \\ c Institut de Mécanique des Fluides de Toulouse, CNRS-Université de Toulouse, 31400 Toulouse, France
}

Keywords:

Thin films

Gas flows

Interfacial flows

Moving mesh

Low-Mach

Long-wave model

\begin{abstract}
A B S T R A C T
The non-linear dynamics of thin liquid films sheared by a laminar gas flow in a channel is investigated. Such a two-layer flow is driven by pressure gradient and possibly by the gravity force. We describe the liquid phase with a long-wave integral model, with the aim to save computational cost with respect to the full Direct Numerical Simulation (DNS) of the Navier-Stokes equations. We derive this long-wave model by the integration of the Navier-Stokes equations over the film thickness, and by an asymptotic expansion up to the first order in terms of a long-wave parameter. These depth-integrated (or shallow water) equations are discretized by means of an augmented system, which holds an evolution equation for the surface tension in order to avoid numerical instabilities of classical upwind and centered schemes. On the other side, we study the gas phase with compressible Navier-Stokes equations, and we discretize them by means of a lowMach scheme, accounting also for moving meshes (ALE). In order to analyze liquidgas interactions, we introduce then a coupling methodology between depth-integrated equations and Navier-Stokes equations. This approach represents a compromise between the two existing methods: the full DNS, and the full long-wave model applied to both phases. In order to validate this approach, we present comparisons with DNS, showing a good agreement of spatio-temporal evolutions of the film thickness and the stress field. Furthermore, interfacial shear stress and pressure gradient evolutions are shown to be in accordance with those provided by two-layer second-order low-dimensional models.
\end{abstract}

\section{Introduction}

Thin liquid films sheared by a laminar gas flow are often encountered in several industrial processes, such as heat and mass transfers in heat pipes, evaporators and distillation columns. Meanwhile, two-layer flows might also be found in the aerospace domain, where the gas is meanly turbulent: pre-filming for injection systems, water ingestion within turboengines, de-icing of aircraft systems, deposition of alumina films over the walls of solid rocket motors.

Because of the development of surface waves, the deformable interface plays a relevant role in the interaction between the two phases. Indeed, experiments [1,2] have proved that the occurrence of waves at the interface considerably amplifies

\footnotetext{
* Corresponding author.

E-mail addresses: gianluca.lavalle@gmail.com (G. Lavalle), vila@insa-toulouse.fr (J.-P. Vila), ghislain.blanchard@math.univ-toulouse.fr (G. Blanchard), claire.laurent@onera.fr (C. Laurent), charru@imft.fr (F. Charru). 
the transfers between the liquid and the gas. Given that these problems are of high complexity, numerical simulations turn to be useful for engineering investigations. There exist several works about DNS applied to film-gas dynamics, that is to say performed by using the Navier-Stokes equations for both the phases. Concerning liquid films in a passive gas atmosphere, we recall the studies of Salamon et al. [3] and Trifonov [4], as well as Ramaswamy et al. [5], who have coupled a finite-element approach with the Arbitrary Lagrangian Eulerian (ALE) method to study temporal and spatial stability of non-linear waves; for two-layer co-current flows, the main works are those of Li et al. [6], Zhang et al. [7] and Frank [8,9], as well as Dietze \& Ruyer-Quil [10] with Gerris (Popinet [11]) and OpenFOAM flow solvers, while Trifonov [12] has investigated counter-current flows.

However, experiments [13-17] have shown that the waves developing on gravity-driven liquid films, or at the liquid-gas interface for channel flows, are much longer than the film thickness. This suggests the introduction of low-dimensional models built on a film parameter $\varepsilon=h / \lambda<<1$ which measures the smallness of the film thickness compared to the wavelength of interfacial waves. Furthermore, the development of low-dimensional models to describe the dynamics of film flows and two-layer flows in general, helps decrease the computational cost with respect to DNS, particularly expensive when analyzing the industrial configurations, such as those previously cited.

These reduced models are mostly based on the earliest works of Benney [18] and Shkadov [19] for falling films down an inclined plane. Benney [18] has developed asymptotic solutions for long-wave disturbances of liquid films subject to gravity. However, the resulting evolution equation fails when studying the behavior of an unstable film far from the instability threshold. On the other hand, Shkadov [19] has developed an integral model of the boundary-layer equations. Despite this model ensures to capture the long-wave interfacial instabilities, it does not assure the right stability threshold. This shortcoming has been then addressed by Ruyer-Quil \& Manneville [20,21], by adding corrections of further orders in $\varepsilon$ to the velocity profile within the liquid film.

In a recent work, Dietze \& Ruyer-Quil [10] have developed a fully reduced model for confined two-layer flows, extending the weighted residual boundary-layer technique (WRIBL) [21] to two phases. This model allows to get rid of the weakness of previous works involving the modelization of two-layer flows [22-25], which are based on the above mentioned studies of Benney and Shkadov. Nevertheless, the use of integral equations for the gas phase restricts the field of application of such a model because the thickness of the gas flow must be much smaller than the wavelength of interfacial waves.

Meanwhile, the WRIBL approach has been also employed by Tseluiko \& Kalliadasis [26] to model liquid films sheared by a counter-current turbulent gas flow. However, given the high gas speed, they have legitimately neglected the film velocity, which in turn becomes essential with comparable film-gas velocities, such as the scenarios treated in this work. Subsequently, Vellingiri et al. [27] have used the same methodology to study co-current flows inside a channel.

Therefore, in this paper we introduce a new model for two-layer channel flows, with the aim to overcome the restrictions of the previously cited works. We couple a low-dimensional model for the liquid phase to compressible Navier-Stokes equations accounting for the gas phase. This methodology, that we call SWANS (Shallow Water ALE Navier-Stokes), is based on the moving mesh technique (ALE) and can be then integrated inside industrial codes, such as the CEDRE platform [28] developed at ONERA. This represents the main reason for having chosen to solve flows at low Mach number with compressible schemes.

When compared to the previously cited works, our model provides the following advantages: the gas thickness is not restricted by the long-wave theory, permitting to study numerous industrial applications; the use of Navier-Stokes equations for the gas phase allows us to have a wide description of the gas fields, namely velocity, shear stress and pressure; the methodology of coupling shallow water model to Navier-Stokes equations represents an intermediate approach between the resolutions typically used for two-layer flows, that are either the fully Navier-Stokes or the fully shallow water approaches; the low-dimensional model used for the liquid phase allows reducing the computational cost compared to DNS. Furthermore, the use of the ALE method represents an originality in the coupling process: so far it has been applied only to gravity-driven falling film or two-phase Navier-Stokes equations (Hirt et al. [29], Chan [30], Pracht [31], Soulaïmani et al. [32] and Ramaswamy et al. [5]). Original from a computational point of view is also the combination of the ALE technique and the low-Mach scheme.

The article is structured as follows: the physical problem is discussed in Section 2; the long-wave film model is described and derived in Section 3; Section 4 shows the coupling strategy between the long-wave film model and Navier-Stokes equations accounting for the gas phase; Section 5 describes the computational approach for the liquid phase and the gas phase, as well as the coupling between the two; Section 6 shows two test-cases and the main results; Section 7 gathers the conclusions of this work.

\section{The general framework}

The problem considered hereby is a two-layer channel flow, as sketched in Fig. 1. The domain is two-dimensional: a thin liquid film on the lower wall of the channel is sheared by a laminar gas flow on the top of it. The two-layer flow is driven by pressure gradient and possibly by the gravity force, through the inclination $\beta$ of the channel. With reference to Fig. $1, H$ denotes the height of the channel, and $h$ the local thickness of the liquid film. Subscripts 1 and 2 refer to liquid and gas phases, respectively.

With this article, our aim is to develop a coupling methodology between depth-integrated equations and Navier-Stokes equations, accounting for liquid and gas phases, respectively. By means of this coupling technique, we want to study the 


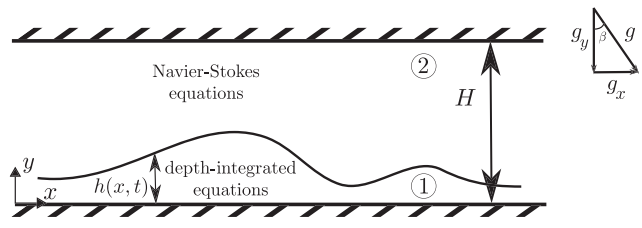

Fig. 1. Sketch of the two-layer channel flow: index 1 refers to the liquid film, while index 2 to the gas phase.

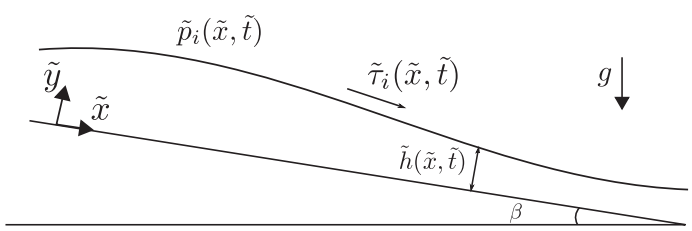

Fig. 2. Sketch of a liquid film subject to gravity and to interfacial shear stress and pressure.

non-linear wave dynamics at the interface between the two phases. We first study the liquid problem, and develop a low-dimensional model for thin liquid films sheared by a gas flow, by means of the long-wave theory. This model is accurate at order one in $\varepsilon$ : it predicts the right linear stability threshold of long waves and is valid in the limit $\varepsilon R e<<1$. In addition, as shown later, space-time thickness variations of the liquid film are assumed to be small, while no assumption is required about the amplitude of the thickness itself. Subsequently, we present how to couple this model to the Navier-Stokes equations in the gas phase.

\section{Liquid film modeling}

With reference to Fig. 2, the governing equations for liquid films down an inclined plane and subject to given interfacial shear stress $\tilde{\tau}_{i}(\tilde{x}, \tilde{t})$ and pressure $\tilde{p}_{i}(\tilde{x}, \tilde{t})$, are the incompressible Navier-Stokes equations:

$$
\rho\left(\partial_{t} \tilde{\mathbf{u}}+\tilde{\mathbf{u}} \cdot \nabla \tilde{\mathbf{u}}\right)=-\nabla \tilde{p}+\rho \mathbf{g}+\mu \nabla^{2} \tilde{\mathbf{u}}, \quad \nabla \cdot \tilde{\mathbf{u}}=0 .
$$

Here the tilde designates dimensional variables, $\nabla$ the nabla operator, $\tilde{\mathbf{u}}=(\tilde{u}, \tilde{v})$ is the film velocity with stream-wise $(\tilde{x})$ and cross-stream $(\tilde{y})$ components, $\tilde{p}$ the pressure, $\mathbf{g}=(g \sin \beta,-g \cos \beta)$ the gravity force, and $v=\mu / \rho$ the kinematic viscosity of the liquid.

At the bottom, where $\tilde{y}=0$, the no-slip condition reads

$$
\tilde{u}=0, \quad \tilde{v}=0 .
$$

At the film surface instead, where $\tilde{y}=\tilde{h}$, boundary conditions state the continuity of tangential and normal stresses, namely

$$
\begin{aligned}
& \tilde{\mathbf{t}} \cdot \tilde{\mathbf{T}}^{(\tilde{n})}=\tilde{\tau}_{i}(\tilde{x}, \tilde{t}), \\
& \tilde{\mathbf{n}} \cdot \tilde{\mathbf{T}}^{(\tilde{n})}=\tilde{p}_{i}(\tilde{x}, \tilde{t})+\gamma \nabla \cdot \tilde{\mathbf{n}},
\end{aligned}
$$

where $\tilde{\mathbf{t}}$ and $\tilde{\mathbf{n}}$ are the tangent and outward normal unit vectors to the interface, $\tilde{\mathbf{T}}^{(\tilde{n})}=\tilde{\Sigma} \cdot \tilde{\mathbf{n}}$ is the stress vector at the interface, while $\tilde{\Sigma}$ is the stress tensor, and $\gamma$ the surface tension. The conditions at the interface are completed by the kinematic condition, which imposes the interface to be a material line, and reads

$$
\partial_{t} \tilde{h}+\left.\tilde{u}\right|_{h} \partial_{x} \tilde{h}=\left.\tilde{v}\right|_{h} .
$$

The system of equations (1) with the corresponding boundary conditions (2)-(5) entirely describes the dynamics of the liquid film of Fig. 2.

In order to identify the dominant terms of the system (1), we work with dimensionless equations by means of dimensionless variables representative of the liquid flow, such as

$$
\begin{array}{lll}
x=\frac{\tilde{x}}{\tilde{h}_{0}}, & y=\frac{\tilde{y}}{\tilde{h}_{0}}, & t=\frac{\tilde{t}}{\tilde{h}_{0} / \tilde{U}_{0}}, \\
u=\frac{\tilde{u}}{\tilde{U}_{0}}, & v=\frac{\tilde{v}}{\tilde{U}_{0}}, & p=\frac{\tilde{p}}{\rho \tilde{U}_{0}^{2}},
\end{array}
$$

Here, $\tilde{h}_{0}$ is the film thickness and $\tilde{U}_{0}$ the average velocity of the uniform flow (alternatively, other quantities might be chosen as characteristic scales).

Furthermore, as already mentioned, waves that develop on the surface of a liquid film at moderate Re are generally long compared to the film thickness. Therefore, the film parameter $\varepsilon<<1$ scales space and time derivatives $\partial_{x, t}$ (with the 
exception of $\partial_{x} p$ given to the pressure scaling), revealing that no assumption must be considered about the amplitude of the thickness itself.

As a matter of fact, for $\varepsilon R e<<1$ the governing equations (1) up to $\mathcal{O}(\varepsilon)$ reduce to the boundary-layer equations. Their dimensionless form reads

$$
\left\{\begin{array}{l}
\partial_{x} u+\partial_{y} v=0 \\
\partial_{t} u+u \partial_{x} u+v \partial_{y} u=-\partial_{x} p+\frac{1}{F r} \sin \beta+\frac{1}{R e} \partial_{y y} u \\
0=-\frac{1}{F r} \cos \beta-\partial_{y} p
\end{array}\right.
$$

where the Reynolds and Froude numbers are defined as

$$
\operatorname{Re}=\frac{\tilde{U}_{0} \tilde{h}_{0}}{v}, \quad F r=\frac{\tilde{U}_{0}^{2}}{g \tilde{h}_{0}} .
$$

The corresponding boundary conditions (2) at the wall, where $y=0$, are

$$
u=0, \quad v=0 .
$$

At the interface, where $y=h$, the continuity of tangential and normal stresses (3)-(4), as well as the kinematic condition (5), become respectively

$$
\begin{aligned}
& \left.p\right|_{h}=p_{i}(x, t)-\frac{1}{W e} \partial_{x x} h, \\
& \left.\partial_{y} u\right|_{h}=\tau_{i}(x, t), \\
& \partial_{t} h+\left.u\right|_{h} \partial_{x} h=\left.v\right|_{h},
\end{aligned}
$$

where We $=\rho \tilde{U}_{0}^{2} \tilde{h}_{0} \gamma^{-1}$ is the Weber number. The system of equations (7) completed by boundary conditions (9) and (10) represents the full dimensionless boundary-layer system of the problem of Fig. 2.

\subsection{Integration over the film thickness}

As already mentioned, we use a long-wave integral model to study the liquid film: the integration of the equations allows us to reduce the degrees of freedom of the system and pass from unknowns $u, v$ and $p$ to the average film thickness $h$ and flow rate $q$. However, before integrating the boundary-layer equations (7), it is suitable to replace the y-momentum equation into the $\mathrm{x}$-momentum through the pressure $p$. This can be achieved by integrating the third of the (7) with the boundary condition (10a), which leads to the hydrostatic pressure field

$$
p(x, y, t)=-\int_{0}^{x} G(x) d x-\frac{1}{W e} \partial_{x x} h+\frac{1}{F r} \cos \beta(h-y) .
$$

With reference to the equation (10a), $-G(x)$ is the interfacial pressure gradient $\partial_{x} p_{i}$ in the gas (alternatively, one can choose the pressure gradient at the bottom). Thus, boundary-layer equations ( 7 ) reduce to a system of two equations, which reads

$$
\left\{\begin{array}{l}
\partial_{x} u+\partial_{y} v=0 \\
\partial_{t} u+u \partial_{x} u+v \partial_{y} u=G-\frac{\cos \beta}{F r} \partial_{x} h+\frac{\sin \beta}{F r}+\frac{1}{R e} \partial_{y y} u+\frac{1}{W e} \partial_{3 x} h
\end{array} .\right.
$$

The integration of these equations over the film thickness is performed by the help of the Leibniz's integration rule and the boundary condition (10c). We find the system

$$
\begin{aligned}
& \partial_{t} h+\partial_{x} q=0, \\
& \partial_{t} q+\partial_{x}\left(\int_{0}^{h} u^{2} d y\right)+\frac{\cos \beta}{F r} h \partial_{x} h=\frac{1}{R e}\left(\Lambda h+\tau_{i}-\left.\partial_{y} u\right|_{0}\right)+\frac{1}{W e} h \partial_{3 x} h,
\end{aligned}
$$

where $q=\int_{0}^{h} u d y$ is the flow rate, $\tau_{w}=\left.\partial_{y} u\right|_{0}$ the wall shear stress and

$$
\Lambda=\frac{\operatorname{Re}}{F r} \sin \beta+\operatorname{Re} G(x)
$$

is the driving force including the stream-wise component of gravity and the pressure gradient. 
These equations couple the film thickness $h$ and the flow rate $q$ (alternatively, y-averaged stream-wise velocity $U=q / h$ ), with the exception of the integral of squared velocity and the wall shear stress. Hence, integrated boundary-layer equations (13) need closure models. In order to close these equations, we provide an asymptotic expansion of the boundary-layer equations (12) with respect to the small parameter $\varepsilon$, and thus obtain velocity and stress fields at $\mathcal{O}(\varepsilon)$.

Recalling that $\partial_{x, t} \sim \varepsilon$ and $v<<u$, the velocity field can be expanded as

$$
\begin{aligned}
& u(x, y, t)=u^{(0)}(x, y, t)+u^{(1)}(x, y, t)+\ldots, \\
& v(x, y, t)=v^{(1)}(x, y, t)+v^{(2)}(x, y, t)+\ldots,
\end{aligned}
$$

where the superscript (0) denotes the leading-order (parallel) flow and (1) denotes order $\varepsilon$ corrections. By doing so, the momentum equation of the system (12) at the leading order simply reads $\Lambda+\partial_{y y} u^{(0)}=0$, where $\Lambda$ is defined in (14). The resulting double integration provides

$$
u^{(0)}(x, y, t)=\tau_{i} y+\Lambda\left(h y-\frac{y^{2}}{2}\right),
$$

thanks to the boundary conditions (10b) and (9). The corresponding flow rate reads

$$
q^{(0)}=\frac{1}{3} \Lambda h^{3}+\frac{1}{2} \tau_{i} h^{2}
$$

The wall shear stress instead is

$$
\tau_{w}^{(0)}=3 \frac{q^{(0)}}{h^{2}}-\frac{1}{2} \tau_{i}
$$

In addition, the cross-stream velocity is given by the continuity equation $\partial_{x} u+\partial_{y} v=0$, as

$$
v^{(1)}=\operatorname{Re} \partial_{x} G \frac{y^{3}}{6}-\left(\partial_{x} \tau_{i}+\Lambda \partial_{x} h+\operatorname{Re} \partial_{x} G h\right) \frac{y^{2}}{2} .
$$

Finally, at $\mathcal{O}(\varepsilon)$, the momentum equation of (12) becomes

$$
\partial_{t} u^{(0)}+u^{(0)} \partial_{x} u^{(0)}+v^{(1)} \partial_{y} u^{(0)}=\frac{1}{W e} \partial_{3 x} h-\frac{\cos \beta}{F r} \partial_{x} h+\frac{1}{R e} \partial_{y y} u^{(1)},
$$

whose double integration gives the velocity profile at order one, namely

$$
\begin{aligned}
u_{1}^{(1)} & =\frac{\operatorname{Re}}{24}(2 h-y)\left(-y^{2}+2 h y+4 h^{2}\right) y\left[\left(\Lambda h+\tau_{i}\right) \Lambda \partial_{x} h-\operatorname{Re} \partial_{t} G\right] \\
& +\frac{\operatorname{Re} 2}{360}\left[\Lambda\left(y^{5}+24 h^{5}+15 h^{2} y^{3}-6 h y^{4}-20 h^{3} y^{2}\right)+3 \tau_{i}\left(-10 h^{4}+5 h y^{3}-2 y^{4}\right)\right] y \partial_{x} G \\
& +\frac{\operatorname{Re}}{24}\left[\left(4 h^{3}-y^{3}\right) \tau_{i}+\left(2 h^{3}+y^{3}-2 h y^{2}\right) \Lambda h\right] y \partial_{x} \tau_{i}-\frac{R e}{6}\left(3 h^{2}-y^{2}\right) y \partial_{t} \tau_{i} \\
& +\frac{\operatorname{Re}}{2}\left(\frac{1}{W e} \partial_{3 x} h-\frac{\cos \beta}{F r} \partial_{x} h\right)(2 h-y) y .
\end{aligned}
$$

The first-order wall shear stress $\tau_{w}^{(1)}=\left.\partial_{y} u^{(1)}\right|_{0}$ instead reads

$$
\begin{aligned}
\tau_{w}{ }^{(1)} & =\frac{1}{3} \Lambda h^{3} \operatorname{Re}\left(\Lambda h+\tau_{i}\right) \partial_{x} h+\frac{1}{60} \operatorname{Re}^{2} h^{4}\left(4 \Lambda h-5 \tau_{i}\right) \partial_{x} G \\
& +\frac{1}{12} \operatorname{Re}^{3}\left(\Lambda h-2 \tau_{i}\right) \partial_{x} \tau_{i}-\frac{1}{3} \operatorname{Re}^{2} h^{3} \partial_{t} G-\frac{1}{2} \operatorname{Re} h^{2} \partial_{t} \tau_{i} .
\end{aligned}
$$

Once velocity and stress fields have been fully computed, system (13) of integrated boundary-layer equations can be finally closed. However, given to the small inertial effects considered in the liquid (the 1.h.s. of $(13 \mathrm{~b})$ is at $\mathcal{O}(\varepsilon)$ ), only the wall shear stress term needs a closure law at order one, whereas the integral of squared velocity requires simply the leading-order parabolic profile (16) (Ruyer-Quil \& Manneville [20,21], Luchini \& Charru [33,34], Kalliadasis et al. [35]). 
Therefore, by replacing the velocity profile (16) into the integral of squared velocity appearing in (13b), and by using the wall shear stress (18) and (22), equations (13) read

$$
\begin{aligned}
& \partial_{t} h+\partial_{x} q=0 \\
& \partial_{t} q+\partial_{x}\left[\frac{6}{5} \frac{q^{2}}{h}+\frac{1}{60} h^{3} \tau_{i}\left(\Lambda h+2 \tau_{i}\right)\right]+\frac{\cos \beta}{F r} h \partial_{x} h=\frac{1}{\operatorname{Re}}\left[\Lambda h+\tau_{i}-\tau_{w}\right]+\frac{1}{W e} h \partial_{3 x} h,
\end{aligned}
$$

where $\tau_{w}=\tau_{w}{ }^{(0)}+\tau_{w}{ }^{(1)}$, given by (18) and (22). One can notice that the closure of the integral of squared velocity follows the gravity-driven film modeling (Shkadov [19]), according to which $\int_{0}^{h} u^{2} d y=6 / 5 q^{2} h^{-1}$. However, for sheared liquid films, the analysis involves an additional term accounting for $\tau_{i}$.

\section{Two-layer flow and liquid-gas boundary conditions}

Recalling that subscripts 1,2 refer to the film and the gas, respectively, we develop here a coupling methodology between the two phases, in order to study the two-layer problem of Fig. 1. Before performing the coupling, we write the system (23) in an another form: we move the $\partial_{x} h$ term of the wall shear stress correction (22) from the r.h.s. to the 1.h.s. of (23b); also, we replace $1 / 5 q^{2} / h$ of the inertial terms in (23b) by using the leading-order flow rate (17), which is valid because the 1.h.s. is $\mathcal{O}(\varepsilon)$. In addition to fulfill the Galilean invariance (Lavalle et al. [36]), this new form keeps the same properties of consistency as (23) and allows us to use the technique of the augmented system for the discrete form, to be shown later. These equations thus read (Lavalle [37])

$$
\begin{aligned}
& \partial_{t} h+\partial_{x} q=0, \\
& \partial_{t} q+\partial_{x}\left(\frac{q^{2}}{h}+P\right)=\frac{1}{R e}\left[\Lambda h-\frac{3 q}{h^{2}}+\frac{3}{2} \tau_{i}-T\right]+\frac{1}{W e} h \partial_{3 x} h,
\end{aligned}
$$

where $P$ is the "pressure" part (in analogy with the Navier-Stokes equations) of the shallow water momentum flux and $T$ derives from the first-order wall shear stress (22):

$$
\begin{aligned}
& P=\frac{2}{225} \Lambda^{2} h^{5}+\frac{1}{15} \Lambda \tau_{i} h^{4}+\frac{1}{12} \tau_{i}{ }^{2} h^{3}+\frac{1}{2} \frac{h^{2}}{F r} \cos \beta \\
& T=\frac{1}{240} h^{3} \operatorname{Re}\left(3 \Lambda h+14 \tau_{i}\right) \partial_{x} \tau_{i}+\operatorname{Re}^{2} h^{4}\left(\frac{3}{175} \Lambda h+\frac{1}{24} \tau_{i}\right) \partial_{x} G+\frac{1}{15} \operatorname{Re}^{2} h^{3} \partial_{t} G+\frac{1}{8} h^{2} \operatorname{Re} \partial_{t} \tau_{i} .
\end{aligned}
$$

We couple the depth-integrated equations (24)-(26) to the compressible Navier-Stokes equations accounting for the gas phase. By using as length, velocity, density and pressure scales the film thickness $\tilde{h}_{0}$, the mean film velocity $\tilde{U}_{0}$ and the uniform gas density $\tilde{\rho}_{2}^{\star}$ and pressure $\tilde{p}_{2}^{\star}$, respectively, the dimensionless form of the Navier-Stokes equations reads

$$
\left\{\begin{array}{l}
\partial_{t} \rho_{2}+\nabla \cdot\left(\rho_{2} \mathbf{u}_{2}\right)=0 \\
\partial_{t}\left(\rho_{2} \mathbf{u}_{2}\right)+\nabla \cdot\left(\rho_{2} \mathbf{u}_{2} \otimes \mathbf{u}_{2}\right)=-\frac{1}{\gamma_{2} M^{2}} \nabla p_{2}+\frac{\rho_{2}}{F r}+\frac{1}{R e_{2}} \nabla \cdot \mathbf{T}_{2}
\end{array}\right.
$$

Here, $\otimes$ is the outer product and $\gamma_{2}$ the heat capacity ratio, while the gas is considered ideal and isothermal resulting in $\tilde{p}=\tilde{\rho} \tilde{R} \tilde{\theta}$, where $\tilde{R}$ is the specific gas constant and $\tilde{\theta}$ the temperature. Since we consider low speed flows, for which $\nabla \cdot \mathbf{u} \simeq 0$, the viscous stress tensor $\mathbf{T}_{2}$ is given by

$$
\mathbf{T}_{2}=2 \mathbf{D}_{2}+\nabla \cdot \mathbf{u}_{2} \mathbf{I} \simeq 2 \mathbf{D}_{2},
$$

where the matrix $\mathbf{D}_{2}=\left(\nabla \mathbf{u}_{2}+\nabla \mathbf{u}_{2}^{T}\right) / 2$ is the strain tensor, whereas $\mathbf{I}$ is the identity matrix. The dimensionless number $F r$ appearing in (27) is the same as in (8), while Mach and Reynolds numbers are defined as

$$
M=\frac{\tilde{U}_{0}}{\tilde{a}^{\star}}, \quad R e_{2}=\frac{\tilde{U}_{0} \tilde{h}_{0}}{\tilde{v}_{2}^{\star}},
$$

where $\tilde{a}^{\star}$ is the reference sound celerity and $\tilde{v}_{2}^{\star}$ the uniform kinematic viscosity of the gas.

The coupling between the two phases takes place at the interface. The gas exerts interfacial shear stress $\tau_{i}$ and pressure gradient $-\tilde{G}$ over the film, which are defined as

$$
\tau_{i}=\left.m \partial_{y} u_{2}\right|_{h}, \quad G=-\left.r \partial_{x} p_{2}\right|_{h},
$$

where $m=\mu_{2} / \mu_{1}$ and $r=\rho_{2} / \rho_{1}$ are the ratio of viscosities and densities, respectively. For what concerns the gas, the top wall is rigid and fixed, in a way that the boundary conditions yield

$$
u_{2}\left(y=\tilde{H} / \tilde{h}_{0}\right)=0, \quad v_{2}\left(y=\tilde{H} / \tilde{h}_{0}\right)=0 .
$$




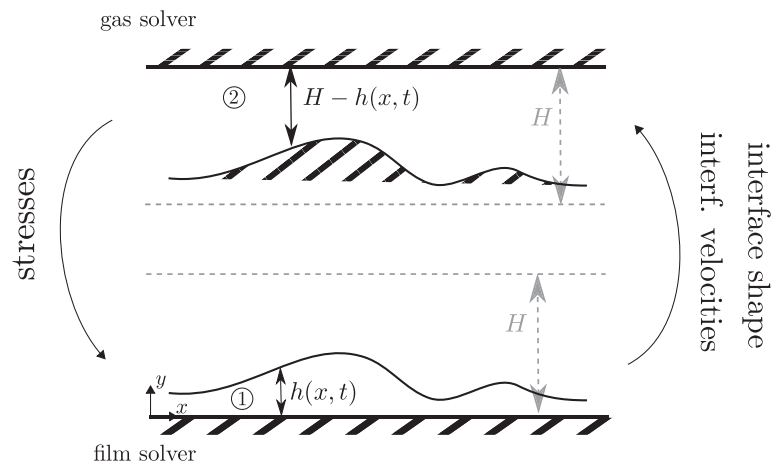

Fig. 3. Sketch of film (bottom) and gas (top) solver geometries. In the background, dashed lines indicate the base configuration, i.e. Fig. 1.

The liquid film transfers to the gas the velocity and the position of the interface: Navier-Stokes equations (27) are thus solved by means of the continuity of velocities and the kinematic condition, namely

$$
\left.u_{2}\right|_{h}=\left.u_{1}\right|_{h},\left.\quad v_{2}\right|_{h}=\left.v_{1}\right|_{h} .
$$

The system is closed by the derivation of $\left.u_{1}\right|_{h}$ through the leading-order velocity profile (16), being the first-order velocity not necessary (see Section 5.3.1). At $y=h$, expression (16) leads to

$$
\left.u_{1}\right|_{h}=\frac{3}{2} \frac{q_{1}}{h}+\frac{1}{4} \tau_{i} h
$$

while $\left.v_{1}\right|_{h}$ is directly obtained by the kinematic condition $\partial_{t} h+\left.u\right|_{h} \partial_{x} h=\left.v\right|_{h}$.

\section{The numerical approach}

In the computational analysis, we treat the two-layer channel flow of Fig. 1 with periodic boundary conditions on left and right sides, while top and bottom sides are rigid walls. Periodic boundary conditions assure that the flow going out of the domain is reinjected at the entrance, verifying the conditions of closed flow. Meanwhile, due to the periodic boundary conditions, the wavelength, rather than the frequency, must be imposed in the performed computational investigations. Initial conditions correspond to the perturbed equilibrium state.

We divide the channel geometry of Fig. 1 into two frameworks, which represent the structures of two distinct solvers to be presented in the following: the bottom of Fig. 3 is referred to the film solver, while the top of Fig. 3 to the gas solver. The film solver allows us to study a sheared liquid film flowing on a flat and rigid wall, and is based on the low-dimensional film model (24)-(26) previously introduced. The domain of resolution of such equations is $\Omega_{1}=\{0 \leq \tilde{x} \leq \tilde{L}\}$, where $\tilde{L}$ is the length of the channel. On the contrary, the gas solver is used to describe a laminar gas bounded on the top by a rigid and fixed wall, and on the bottom by a liquid film, which is modeled as a wavy wall with a certain motion. The gas solver works with equations (27) and accounts for moving meshes. The domain of resolution of the gas equations is $\Omega_{2}(\tilde{t})=\{0 \leq \tilde{x} \leq \tilde{L} ; \tilde{h}(\tilde{x}, \tilde{t}) \leq \tilde{y} \leq \tilde{H}\}$. Furthermore, as highlighted in Fig. 3, the two solvers exchange informations at the interface. Particularly, following the previous section, we choose to provide interfacial shear stress and pressure gradient from the gas solver to the film, while the film solver returns to the gas shape and velocity of the interface. This data exchange permits to model the interface and all mutual effects between the liquid and the gas.

This choice of coupling methodology is coherent with the fact that the long-wave model (24) computes film thickness $h$ and flow rate $q$, which are directly transferred to the gas solver without further manipulations, except for recovering the interfacial velocity (33). However, other choices of liquid-gas coupling are possible. For example, we refer to Habchi et al. [38], who have studied liquid-gas industrial problems by using an order-zero film model for the liquid phase, thus neglecting the correction at order one of the wall shear stress, as well as the retro-action of the liquid given by the deformation of the interface; we also refer to the aforementioned work of Tseluiko \& Kalliadasis [26] for counter-current turbulent flows over a wavy liquid film.

Another interesting feature of our coupling methodology concerns the surface tension discretization. Indeed, given that the film solver transfers to the gas the position of the interface by means of the ALE method, the surface tension has not to be implemented into the gas solver. The gas can see the effects of the surface tension by the deformation of the interface, which is computed at each time step by the film solver taking into account the capillary effects, in accordance with the hydrostatic pressure (11). This allows to avoid the discretization of the surface tension term in the Navier-Stokes analysis. As a consequence, if the capillary characteristic time is the smallest, we can get greater time steps in the computational investigations, compared to the classical full DNS.

However, it must be stated that the gas feels the stresses of the liquid by an indirect way only, i.e. through the transfer of the boundary and the interfacial velocities. A consequence of this might be that the stresses from the liquid are neglected: 
the answer to this issue can be addressed by testing the coupling methodology to the case of two layers of comparable viscosities, which this work does not. We leave thus this matter to future studies. Secondly, it is worthwhile to discuss about the consequences of the long-wave model (24) on the coupling technique. Indeed, the long-wave model developed in Section 3 is based on the assumption that the shear stress $\tau_{i}$ and the pressure gradient $-G$ are bounded for coherence of the asymptotic analysis. This leads to limit the functionality of SWANS to cases where the gas velocity does not reach extremely high values, i.e. when the film becomes excessively thin and atomizes.

This section is structured as: firstly we show the numerical schemes of the film solver (Section 5.1) and the gas solver (Section 5.2), secondly we discuss the numerical coupling between them (Section 5.3).

\subsection{Discrete one-layer depth-integrated equations for the film solver}

We build the film solver (bottom of Fig. 3) by discretization of the depth-integrated equations (24). Since the long-wave model is based on integrated variables, in the numerical approach one single cell in the vertical direction is sufficient to compute the film.

Following the work of Noble \& Vila [39] formulated for the Euler-Korteweg system, we have discretized depth-integrated equations (24) through an augmented system. This method consists in reducing the order of the system by adding one evolution equation for the surface tension, as shown below. This need arises from the discretization of the surface tension which involves third derivatives, i.e. $\partial_{3 x} h$, and for the consequent stability of difference approximation schemes.

Therefore, we have taken the approach of Noble \& Vila [39] and extended it to the depth-integrated equations (24), describing liquid films driven by shear stress and pressure gradient. If one introduces the quantity $w=\sqrt{\sigma} \partial_{x} h / \sqrt{h}$, where $\sigma=W e^{-1}$ is the capillary coefficient of the integrated equations, system (24) takes the form

$$
\begin{aligned}
& \partial_{t} h+\partial_{x}(h U)=0, \\
& \partial_{t}(h U)+\partial_{x}\left(h U^{2}+P\right)=\frac{1}{R e}\left[\Lambda h-\frac{3 U}{h}+\frac{3}{2} \tau_{i}-T\right]+\partial_{x}\left(\varphi(h) \partial_{x} w\right), \\
& \partial_{t}(h w)+\partial_{x}(h U w)=-\partial_{x}\left(\varphi(h) \partial_{x} U\right),
\end{aligned}
$$

where $U=q / h$ and $\varphi(h)=h^{3 / 2} \sqrt{\sigma}$. Equation (36) has been obtained after multiplication of the continuity equation by $\sqrt{h \sigma}$ and subsequent derivation with respect to $x$. It is worthwhile to mention that developing the term $\partial_{x}\left(\varphi(h) \partial_{x} w\right)$ into the equation (35), we exactly get the surface tension term of system (24). As a matter of fact, thanks to the evolution equation for $w$, the resulting system thus contains only second derivatives in $x$.

Following Noble \& Vila [39], system (34)-(36) can be written in the conservative form

$$
\partial_{t} v+\partial_{x} f(v)=s(v)+\partial_{x}\left(B(h) \partial_{x} z\right)
$$

where $v=(h, h U, h w)^{T}$ is the conserved variable vector of depth-integrated equations and $f(v)=\left(h U, h U^{2}+P, h U w\right)^{T}$ the corresponding flux. Vector $s(v)$, matrix $B(h)$ and the product $B(h) \partial_{x} z$ are defined as

$$
s(v)=\left\{\begin{array}{c}
0 \\
\frac{1}{R e}\left[\Lambda h-\frac{3 U}{h}+\frac{3}{2} \tau_{i}-T\right] \\
0
\end{array}\right\}, \quad B(h)=\left(\begin{array}{ccc}
0 & 0 & 0 \\
0 & 0 & \varphi \\
0 & -\varphi & 0
\end{array}\right), \quad B(h) \partial_{x} z=\left\{\begin{array}{c}
0 \\
\varphi \partial_{x} w \\
-\varphi \partial_{x} U
\end{array}\right\},
$$

where $z=\nabla_{v} E(v)=\left(\left(U^{2} / 2+w^{2} / 2\right)+F^{\prime}, U, w\right)$ if we define the total energy as the sum of kinetic and thermodynamic free energies, namely $E=h\left(U^{2} / 2+w^{2} / 2\right)+F(h)$. Note that function $F(h)$ does not enter in the equation (37) given that the first column of $B(h)$ contains all zeros.

\subsubsection{Spatio-temporal discretization schemes}

Discrete equations of the film solver have been obtained by means of a second-order accurate space discretization, and the Rusanov flux for the approximate Riemann solver. Indeed, only a few numerical schemes applied to equations (34)-(36) are found to be entropy stable, in the sense that corresponding difference approximations dissipate the energy, we refer to [39] for further details. Time discretization is computed through the Heun's method, which can be seen as an improved Euler's method or a two-step Runge-Kutta method. If we define $v_{i}^{n}$ as the average value of $v$ at time $n$ within the cell $i$, namely

$$
v_{i}^{n}=\frac{1}{\Delta x} \int_{x_{i-1 / 2}}^{x_{i+1 / 2}} v^{n}(x) d x
$$

and similarly for $s_{i}^{n}$, the integration of equations (37) over the cell $i$ and the time interval $(n, n+1)$ leads to

$$
v_{i}^{n+1 / 2}=v_{i}^{n}-\frac{\Delta t}{\Delta x}\left(f_{i+1 / 2}^{n}-f_{i-1 / 2}^{n}\right)+\Delta t s_{i}^{n}+\frac{\Delta t}{\Delta x^{2}}\left[B_{i+1 / 2}^{n}\left(z_{i+1}^{n}-z_{i}^{n}\right)-B_{i-1 / 2}^{n}\left(z_{i}^{n}-z_{i-1}^{n}\right)\right]
$$


and

$$
\begin{aligned}
v_{i}^{n+1} & =\frac{v_{i}^{n}+v_{i}^{n+1 / 2}}{2}-\frac{\Delta t}{\Delta x} \frac{f_{i+1 / 2}^{n+1 / 2}-f_{i-1 / 2}^{n+1 / 2}}{2} \\
& +\frac{\Delta t}{2}\left[s_{i}^{n+1 / 2}+\frac{1}{\Delta x^{2}}\left(B_{i+1 / 2}^{n+1 / 2}\left(z_{i+1}^{n+1 / 2}-z_{i}^{n+1 / 2}\right)-B_{i-1 / 2}^{n+1 / 2}\left(z_{i}^{n+1 / 2}-z_{i-1}^{n+1 / 2}\right)\right)\right],
\end{aligned}
$$

where superscript $n+1 / 2$ refers to quantities evaluated at the first step of Heun's method. Indices $i, i+1, i-1$ refer to central, right and left cells respectively, while $i+1 / 2$ and $i-1 / 2$ specify interface values, where flux $f$ and matrix $B$, as well as derivatives $\partial_{x} z$, must be evaluated. The discrete quantity $f_{i+1 / 2}^{n}$ is solved with the Rusanov scheme, which is demonstrated to be entropy stable for the Euler-Korteweg equation, see [39]. Omitting the superscript $n$, Rusanov flux yields (Toro [40])

$$
f_{i+1 / 2}=\frac{f\left(v_{i+1 / 2}^{+}\right)+f\left(v_{i+1 / 2}^{-}\right)}{2}-\max _{i+1 / 2^{ \pm}}[U+e] \frac{v_{i+1 / 2}^{+}-v_{i+1 / 2}^{-}}{2},
$$

where $U=v_{y} / v_{x}$ and $e^{2}=d P / d h$ is the characteristic velocity, with $P$ "pressure" term defined in (25). Quantities $v_{i+1 / 2}^{+}$ and $v_{i+1 / 2}^{-}$are the right and left states across the interface in $i+1 / 2$, respectively, and have been found through a MUSCL space discretization. Furthermore, $\left(z_{i+1}^{n}-z_{i}^{n}\right) / \Delta x$ approximates $\partial_{x} z^{n}$ at the interface $i+1 / 2$, where $z_{i}^{n}$ represents $z$ within the cell $i$ at time $n$. In order to write the matrix $B$ at the interface, we use a central discretization, namely $\varphi_{i+1 / 2}=$ $\sqrt{\sigma}\left(\left(h_{i+1}+h_{i}\right) / 2\right)^{3 / 2}$ from the definition of $\varphi$ given in (36).

Recalling the momentum equation (35) of the augmented system of depth-integrated equations, the source term contains space and time derivatives of interfacial shear stress $\tau_{i}$ and pressure gradient $-G$ provided by the gas to the liquid film, through the term $T$ defined in (26). Space derivatives have been discretized by means of central difference approximations, namely

$$
\left(\partial_{x} \tau_{i}\right)_{i}^{n}=\frac{\left(\tau_{i}\right)_{i+1}^{n}-\left(\tau_{i}\right)_{i-1}^{n}}{2 \Delta x}, \quad\left(\partial_{x} G\right)_{i}^{n}=\frac{G_{i+1}^{n}-G_{i-1}^{n}}{2 \Delta x},
$$

while respective time derivatives have been approximated with a backward discretization, that is

$$
\left(\partial_{t} \tau_{i}\right)_{i}^{n}=\frac{\left(\tau_{i}\right)_{i}^{n}-\left(\tau_{i}\right)_{i}^{n-1}}{\Delta t}, \quad\left(\partial_{t} G\right)_{i}^{n}=\frac{G_{i}^{n}-G_{i}^{n-1}}{\Delta t} .
$$

\subsubsection{Stability of the scheme for the film solver}

The characteristic velocity $e^{2}=\frac{2}{45} \Lambda^{2} h^{4}+\frac{4}{15} \Lambda \tau_{i} h^{3}+\frac{1}{4} \tau_{i}^{2} h^{2}+\frac{h}{\mathrm{Fr}} \cos \beta$ must be positive in order to have hyperbolic equations (Whitham [41]). Particularly, all terms of $e^{2}$ are always positive, with the exception of $\Lambda \tau_{i}$ : as a consequence, the sign of $e^{2}$ has to be always verified a posteriori. For example, Fig. 4 shows the time evolution of minimum normalized $e^{2}$ corresponding to the test to be analyzed in Section 6: an air-water system flowing in a confined channel with $R_{1}=4.48$ and $\operatorname{Re}_{2}=36.8$. It is shown that in this configuration $e^{2}$ is always positive and the equations are thus hyperbolic.

For what concerns the Courant-Friedrichs-Lewy (CFL) condition of the scheme, the non-linear stability provides $\Delta t \Delta x^{-2}<$ const., the constant value depending from the initial data, for first-order space discretizations (Corollary 3.4 of [39]). Nevertheless, we use a second-order space discretization, for which a necessary linear stability condition is (Corollary 2.6 of [39] for Lax-Friedrichs scheme)

$$
\frac{\Delta t}{\Delta x}\left(U+\sqrt{e^{2}+\frac{2 \sigma}{\Delta x^{2}}}\right) \leq 1 .
$$

\subsection{Discrete compressible Navier-Stokes equations for the gas solver}

The system of Navier-Stokes equations (27) has been discretized with a first-order spatio-temporal discretization by using a low-Mach scheme in addition to the ALE technique. The low-Mach scheme, based on the work of Grenier et al. [42], allows solving compressible flows at low speeds, where classical approximate Riemann solvers are very dissipative, given that the flow velocity is much smaller than the sound celerity.

The ALE technique is instead used to couple the low-Mach scheme with the discrete depth-integrated equations (34)-(36) for the liquid phase, and will be detailed later.

Before the analysis of the discrete equations and the low-Mach scheme, we show how the Navier-Stokes equations are modified by the node motion of the grid. Recalling the equations (27), this system can be written as (omitting the subscript 2)

$$
L_{\mathbf{u}}=-\nabla \cdot \Sigma^{E}+R e^{-1} \nabla \cdot \mathbf{T}+\mathbf{F},
$$




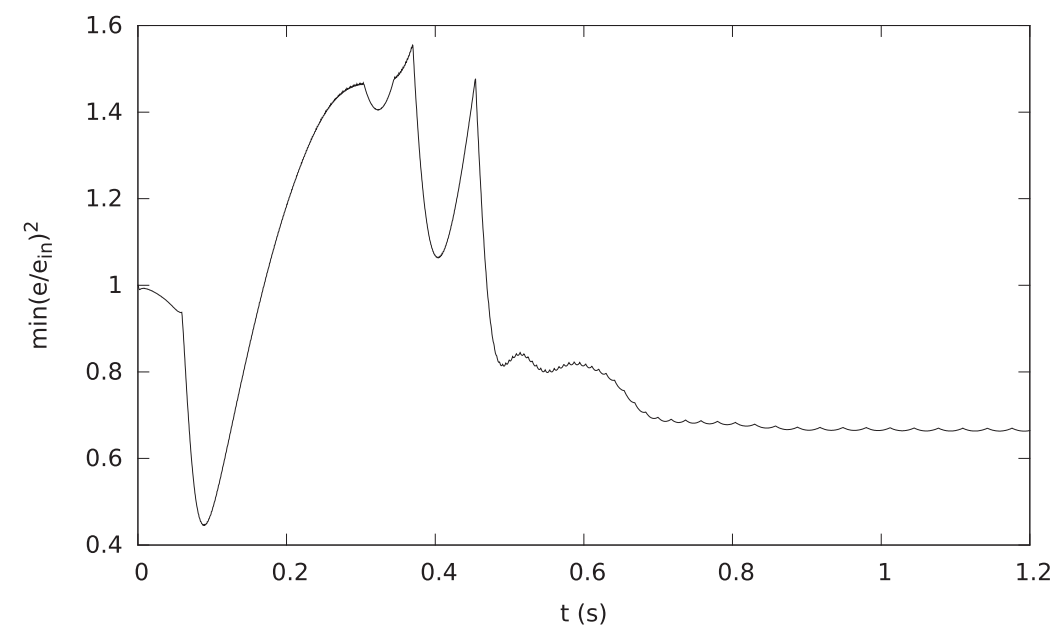

Fig. 4. Time evolution of the minimum characteristic velocity $e^{2}$ normalized with the initial value $e_{\text {in }}=2.5 \cdot 10^{-4} \mathrm{~m}^{2} \mathrm{~s}^{-2}$. Two-layer flow with dimensionless numbers: $R e_{1}=4.48, R e_{2}=36.8, F r=0.93, W e=0.002$, and geometry given by $\tilde{H}=0.39 \mathrm{~mm}$ and $\lambda=26 \mathrm{~mm}$. After $0.7 \mathrm{~s}$ periodic waves appear.

where $L_{\mathbf{u}}=\partial_{t} \phi+\nabla \cdot(\phi \otimes \mathbf{u})$, and $\mathbf{F}=\rho F^{-1}\left(g_{x}, g_{y}\right)^{T}$. The vector $\phi=(\rho, \rho u, \rho v)^{T}$ is the conserved variable vector, while $\Sigma^{E}$ is the "pressure contribution" of the stress tensor $\Sigma=-\Sigma^{E}+\mathbf{T}$. These are defined as

$$
\boldsymbol{\Sigma}_{x}^{E}=\left\{\begin{array}{c}
0 \\
\Pi \\
0
\end{array}\right\}, \quad \boldsymbol{\Sigma}_{y}^{E}=\left\{\begin{array}{c}
0 \\
0 \\
\Pi
\end{array}\right\}, \quad \mathbf{T}_{x}=\left\{\begin{array}{c}
0 \\
T_{x x} \\
T_{x y}
\end{array}\right\}, \quad \mathbf{T}_{y}=\left\{\begin{array}{c}
0 \\
T_{x y} \\
T_{y y}
\end{array}\right\},
$$

where $\Pi=\gamma_{2}^{-1} M^{-2} p$. When the grid moves, a mesh velocity has to be involved into the Navier-Stokes equations, and we introduce a generic mesh velocity $\hat{\mathbf{u}}$ for each edge, to be defined later, corresponding to the node motion. Therefore, the effect of the arbitrary motion of the computational mesh leads to rewrite equations (46) as

$$
L_{\hat{\mathbf{u}}}=-\nabla \cdot\left[\boldsymbol{\Sigma}^{E}+\phi \otimes(\mathbf{u}-\hat{\mathbf{u}})\right]+R e^{-1} \nabla \cdot \mathbf{T}+\mathbf{F},
$$

where $L_{\hat{\mathbf{u}}}=\partial_{t} \phi+\nabla \cdot(\phi \otimes \hat{\mathbf{u}})$. Finally, the motion of the grid modifies only the inviscid side of the equations.

\subsubsection{Low-Mach scheme description}

In order to develop the above mentioned low-Mach scheme and discretize equations (48), Fig. 5 shows the notation used in the following for the development of discrete equations: $K$ designates the cell, Ke its neighborhood cells and $\mathbf{n}_{e, K}$ the outward normal corresponding to the edge $e$. Therefore, following Grenier et al. [42], equations (48) are discretized as

$$
\begin{gathered}
\rho_{K}^{n+1}=\frac{m_{K}^{n}}{m_{K}^{n+1}} \rho_{K}^{n}-\frac{\Delta t}{m_{K}^{n+1}} \sum_{e \in \partial K}\left(l^{-} \rho_{K e}^{n+1}+l^{+} \rho_{K}^{n+1}\right) m_{e}^{n+1} \\
\rho_{K}^{n+1} \mathbf{u}_{K}^{n+1}=\frac{m_{K}^{n}}{m_{K}^{n+1}} \rho_{K}^{n} \mathbf{u}_{K}^{n}+\frac{\Delta t}{m_{K}^{n+1}} \sum_{e \in \partial K}\left(l^{-} \rho_{K e}^{n+1} \mathbf{u}_{K e}^{n}+l^{+} \rho_{K}^{n+1} \mathbf{u}_{K}^{n}+\Pi_{e}^{n+1} \mathbf{n}_{e, K}^{n+1}\right) m_{e}^{n+1} \\
+\Delta t \frac{m_{K}^{n}}{m_{K}^{n+1}} \mathbf{F}_{K}^{n+1}+\frac{R e^{-1}}{m_{K}^{n+1}} \sum_{e \in \partial K} \mathbf{T}_{e}^{n} \cdot \mathbf{n}_{e, K}^{n+1} m_{e}^{n+1}
\end{gathered}
$$

where $\partial K$ is the cell contour, $m_{K}$ the cell surface and $m_{e}$ the length of each edge. Superscripts $n$ and $n+1$ refer to the time step. We also define

$$
\begin{aligned}
& l^{-}=\min \left[\mathbf{w}_{e}^{n} \cdot \mathbf{n}_{e, K}^{n+1}, 0\right]-\Gamma_{e} \max \left[\Pi_{K e}^{n+1}-\Pi_{K}^{n+1}, 0\right], \\
& l^{+}=\max \left[\mathbf{w}_{e}^{n} \cdot \mathbf{n}_{e, K}^{n+1}, 0\right]-\Gamma_{e} \min \left[\Pi_{K e}^{n+1}-\Pi_{K}^{n+1}, 0\right], \\
& \mathbf{w}_{e}^{n}=\frac{1}{2}\left(\mathbf{u}_{K}^{n}+\mathbf{u}_{K e}^{n}\right)-\hat{\mathbf{u}}_{e}^{n}, \\
& \Pi_{e}^{n+1}=\frac{1}{2}\left(\Pi_{K}^{n+1}+\Pi_{K e}^{n+1}\right) .
\end{aligned}
$$

Parameter $\Gamma_{e}$ is instead a positive coefficient to be adjusted for the stability of the scheme (see Section 5.2.2). The velocity $\mathbf{w}_{e}^{n}$ defined on the edge $e$ at time $n$ takes into account also the ALE velocity $\hat{\mathbf{u}}_{e}^{n}$, to be defined later. Furthermore, the pressure $p$ can be written as a function of the density $\rho$ by linearizing the equation of state around the base state. 


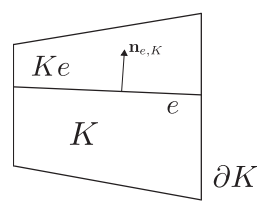

Fig. 5. Notations for the low-Mach scheme used in this work.

Some remarks must be done about the equations (49)-(51). As already stated, those are based on the work of Grenier et al. [42] for two-phase flows. However, we highlight the main differences: firstly, in this work the equations (49)-(51) are used for a single phase, that is the gas. In addition, we have extended the work of Grenier et al. [42] by adding the possibility of moving meshes. We can notice that the moving mesh manifests itself by the presence of the edge velocity $\hat{\mathbf{u}}_{e}^{n}$ in (51c). As a consequence, both the edge length and cell surface are different from those at the previous time step, which makes the difference between $m_{e}^{n}$ and $m_{K}^{n}$ with $m_{e}^{n+1}$ and $m_{K}^{n+1}$, respectively. We also recall that in the ALE framework we have $\frac{d}{d t}(m(t))=\nabla \cdot(\hat{\mathbf{u}})$.

For what concerns the viscous stress tensor, given the definition (28), its discretization turns into the reconstruction of the velocity gradient on every edge starting from the average velocity in the center of each cell. We will not detail here the method used for non-orthogonal meshes, which is based on the diamond cell strategy, and refer to Coudière et al. [43].

\subsubsection{Energy stability of the gas solver}

The stability of the low-Mach scheme is analyzed as in Grenier et al. [42], and we get that the total free energy of the flow is a time decreasing function. We define the total free energy of the system as $E=\frac{1}{2} \rho\|\mathbf{u}\|^{2}+\Phi$, with $\Phi=\rho \int_{\varrho_{*}}^{\varrho} \frac{p(r)}{r^{2}} d r$ corresponding to the barotropic pressure. Following the same calculations as in the stability proof of [42], we write the discrete energy equation as (omitting viscous terms and gravity)

$$
\begin{aligned}
& m_{K}^{n+1} E_{K}^{n+1}-m_{K}^{n} E_{K}^{n}+\Delta t \sum_{e \in \partial K}\left[\left(\mathbf{w}_{e}^{n} \cdot \mathbf{n}_{e, K}^{n+1}\right)^{+} E_{K}^{n+1}+\left(\mathbf{w}_{e}^{n} \cdot \mathbf{n}_{e, K}^{n+1}\right)^{-} \rho_{K e}^{n+1} E_{K e}^{n+1}\right] m_{e}^{n+1} \\
& +\frac{\Delta t}{2} \sum_{e \in \partial K}\left[\left(\mathbf{u}_{K}^{n} \Pi_{K e}^{n+1}+\Pi_{K}^{n+1} \mathbf{u}_{K e}^{n}\right) \cdot \mathbf{n}_{e, K}^{n+1} m_{e}^{n+1}-\Gamma_{e}\left(\Pi_{K e}^{n+1}+\Pi_{K}^{n+1}\right)\left(\Pi_{K e}^{n+1}-\Pi_{K}^{n+1}\right) m_{e}^{n+1}\right] \\
& =m_{K}^{n+1}\left(Q_{K}^{n}+R_{K}^{n}\right)+\Delta t S_{K}^{n} .
\end{aligned}
$$

Here, superscripts,+- denote $\max [\cdot, 0], \min [\cdot, 0]$ respectively. Defining $\Phi^{\prime}=\kappa$, by a Taylor expansion of $\Phi$ there exists a certain $\theta \in[0,1]$ such that

$$
\Phi\left(\rho_{K}^{n+1}\right)-\Phi\left(\rho_{K}^{n}\right)=\left(\rho_{K}^{n}-\rho_{K}^{n}\right) \kappa_{K}^{n+1}-\frac{1}{2} \widetilde{\kappa}_{K}^{\prime n+1}\left(\rho_{K}^{n}-\rho_{K}^{n}\right)^{2},
$$

where $\widetilde{\kappa}_{K}^{\prime n+1}=\kappa^{\prime}\left((1-\theta) \rho_{K}^{n}+\theta \rho_{K}^{n+1}\right)$, and similarly for $\widetilde{\kappa}_{e}^{\prime n+1}$. Note also that $\kappa^{\prime}=a^{2} \rho^{-1}>0$.

$$
\begin{aligned}
& m_{K}^{n+1} Q_{K}^{n}=m_{K}^{n+1} \frac{1}{2} \rho_{K}^{n+1}\left\|\mathbf{u}_{K}^{n+1}-\mathbf{u}_{K}^{n}\right\|^{2}+\Delta t \sum_{e \in \partial K}\left(\mathbf{w}_{e}^{n} \cdot \mathbf{n}_{e, K}^{n+1}\right)^{-} \rho_{K e}^{n+1} \frac{1}{2}\left\|\mathbf{u}_{K e}^{n}-\mathbf{u}_{K}^{n}\right\|^{2} m_{e}^{n+1} \\
&-\frac{\Delta t}{2} \sum_{e \in \partial K} \Gamma_{e}\left(\Pi_{K e}^{n+1}-\Pi_{K}^{n+1}\right)^{2} m_{e}^{n+1}, \\
& m_{K}^{n+1} R_{K}^{n}=-\frac{1}{2} m_{K}^{n} \widetilde{\kappa}_{K}^{n+1}\left(\rho_{K}^{n+1}-\rho_{K}^{n}\right)^{2}+\frac{1}{2} \Delta t \sum_{e \in \partial K}\left(\mathbf{w}_{e}^{n} \cdot \mathbf{n}_{e, K}^{n+1}\right)^{-} \widetilde{\kappa}_{e}^{n+1}\left(\rho_{K}^{n+1}-\rho_{K e}^{n+1}\right)^{2} m_{e}^{n+1}, \\
& S_{K}^{n}=-\Pi_{K}^{n+1}\left(\left(\frac{m_{K}^{n+1}-m_{K}^{n}}{\Delta t}\right)-\sum_{e \in \partial K}\left(\hat{\mathbf{u}}_{e}^{n} \cdot \mathbf{n}_{e, K}^{n+1}\right) m_{e}^{n+1}\right) .
\end{aligned}
$$

Using the Cauchy-Schwarz inequality, the term $Q_{K}^{n}$ can be estimated as

$$
\begin{aligned}
& Q_{K}^{n} \leq \frac{\Delta t}{2 m_{K}^{n+1}}\left(\sum_{e \in \partial K}\left(\frac{\Delta t}{2 m_{K}^{n+1} \rho_{K}^{n+1}} m_{\partial K}^{n+1}-\Gamma_{e}\right)\left(\Pi_{K e}^{n+1}-\Pi_{K}^{n+1}\right)^{2} m_{e}^{n+1}\right)+(1- \\
& \left.\frac{2 \Delta t}{m_{K}^{n+1} \rho_{K}^{n+1}}\left(\sum_{e \in \partial K}\left(-\mathbf{w}_{e}^{n} \cdot \mathbf{n}_{e, K}^{n+1}\right)^{-} \rho_{K e}^{n+1} m_{e}^{n+1}\right)\right)\left(\frac{\Delta t}{m_{K}^{n+1}} \sum_{e \in \partial K}\left(\mathbf{w}_{e}^{n} \cdot \mathbf{n}_{e, K}^{n+1}\right)^{-} \rho_{K e}^{n+1} \frac{1}{2}\left\|\mathbf{u}_{K e}^{n}-\mathbf{u}_{K}^{n}\right\|^{2} m_{e}^{n+1}\right) .
\end{aligned}
$$

We notice that $R_{K}^{n}$ in (53b) is negative because $\left(\mathbf{w}_{e}^{n} \cdot \mathbf{n}_{e, K}^{n+1}\right)^{-} \leq 0$, while $Q_{K}^{n}$ is negative if the following conditions are satisfied: 


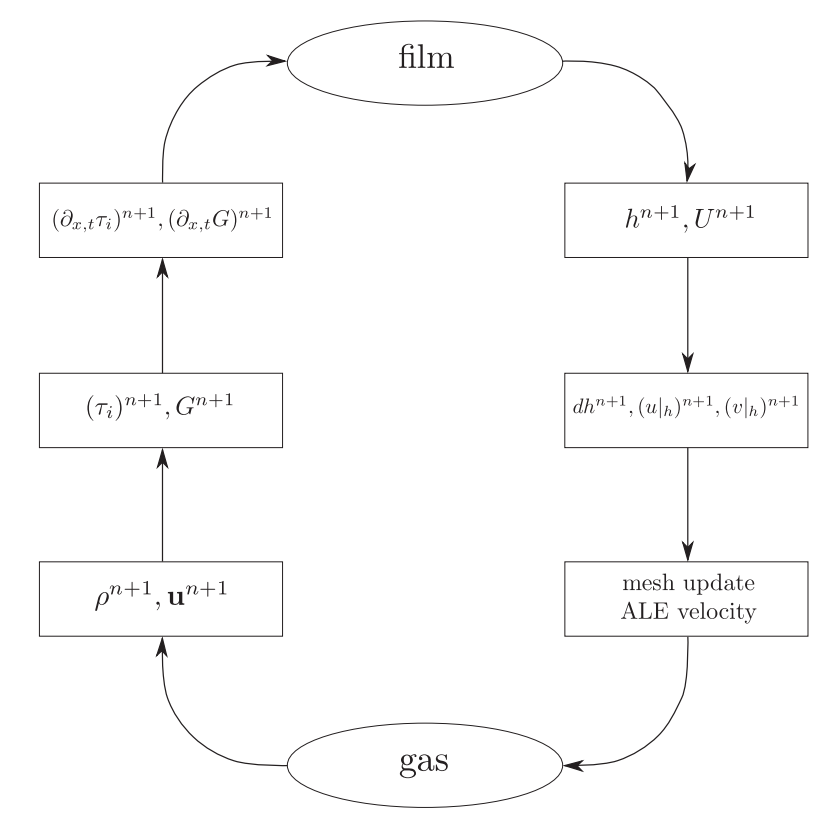

Fig. 6. Sketch of the coupling methodology and time progress $n \longrightarrow n+1$.

$$
\Delta t \leq \frac{\Delta m}{2 l_{K}}, \quad \Gamma_{e} \geq \Delta t \max \left[\frac{m_{\partial K}^{n+1}}{2 \rho_{K}^{n+1} m_{K}^{n+1}}, \frac{m_{\partial K e}^{n+1}}{2 \rho_{K e}^{n+1} m_{K e}^{n+1}}\right],
$$

where $l_{K}$ and $\Delta m$ are defined as

$$
l_{K}=\frac{1}{\rho_{K}^{n+1} m_{\partial K}^{n+1}} \sum_{e \in \partial K}\left|l^{-}\right| \rho_{K e}^{n+1} m_{e}^{n+1}, \quad \Delta m=\frac{m_{K}^{n+1}}{m_{\partial K}^{n+1}},
$$

where $l^{-}$is in (51a). One notices that the discrete mass conservation equation (49) is implicit, permitting to relax the CFL condition (54) of the scheme, because the stability condition does not contain the sound celerity. Concerning the term $S_{K}^{n}$ in (53c), it can be considered zero if fulfills exactly the corresponding discrete geometric conservation (DCGL), see Farhat et al. [44]. Indeed, we notice that (53c) is a discrete version of $\frac{d}{d t}(m(t))=\nabla \cdot(\hat{\mathbf{u}})$ which is the basic rule for mesh evolution in the ALE framework. In this case we have thus proven that

$$
\sum_{K} m_{K}^{n+1} E_{K}^{n+1} \leq \sum_{K} m_{K}^{n} E_{K}^{n}
$$

If we only have first order consistency of the geometric conservation, rather than exact discrete geometric conservation, we get that $\frac{d}{d t}(m(t))-\nabla \cdot(\hat{\mathbf{u}})=\mathcal{O}\left(m_{K}^{n+1} \Delta t\right)$, and we keep a control of the total energy in $e^{C t}$ through Gronwall's lemma. We refer to Donea et al. [45] for additional references about DCGL and to Lanson \& Vila [46] for stability proof of ALE type schemes for conservation laws.

\subsection{Coupling approach development}

After the description of film and gas solvers, we discuss the numerical coupling between the two. Fig. 6 summarizes all steps of the coupling process in the range $(n, n+1)$ :

1. the film solver computes film thickness $h$ and average velocity $U$ at time level $n+1$. By doing so, equation (35) shows that interfacial shear stress $\tau_{i}$ and pressure gradient $-G$, as well as their respective spatio-temporal derivatives must be known. These are given either from the previous computation at time $n$ or from the initial conditions at time $t=0$;

2. film thickness $h^{n+1}$ and average velocity $U^{n+1}$ can be then used to evaluate the velocity field at the interface, namely $\left(\left.u\right|_{h}\right)^{n+1}$ and $\left(\left.v\right|_{h}\right)^{n+1}$ (as shown in Section 4), and the displacement of the interface with respect to the previous time step;

3. subsequently, the position of the interface at time $n+1$ can be manipulated to update the mesh of the gas solver. The bottom cell of the grid moves exactly as the interface, while the motion of all other nodes is submitted to a mesh-update law, to be discussed later;

4. the displacement of every single node allows evaluating the ALE velocity $\hat{\mathbf{u}}$ corresponding to each edge; 
5. the gas solver takes as input the interfacial velocities, the updated mesh and the velocity $\hat{\mathbf{u}}$ to compute density and velocity fields at time step $n+1$ (low-Mach scheme previously discussed);

6. finally, interfacial shear stress $\tau_{i}^{n+1}$ and pressure gradient $-G^{n+1}$ can be computed, as well as their respective spatiotemporal derivatives by means of central difference and backward discretization (see (43) and (44)).

This coupling methodology applies a first-order time discretization and is explicit, in a way that the gas solver adopts at time level $n$ the output provided by the film evaluated at the step $n+1$. As a consequence, the time step is imposed by the minimum between the two solvers. In certain configurations, the viscous time step $t_{v} \simeq \Delta y^{2} / v_{g}$ represents the lower limit, since we treat the discrete viscous term explicitly. Otherwise, the time step is imposed by the film stability condition (see (45)), in particular when we refine in the $x$-direction.

The main effect of the time-explicit coupling technique is that the time-scale ratio in the gas and in the liquid must be high. In other words, the film cannot capture the phenomena faster than its time scaling $\tilde{h}_{0} / \tilde{U}_{0}$. For example, this would be the case of the acoustic effects in the gas, although in the performed tests those are negligible, as shown later (Section 6.1.3).

\subsubsection{Film to gas coupling}

As already stated, the film solver takes interfacial shear stress $\tau_{i}$ and pressure gradient $-G$ as input for the computation of film thickness $h$ and average velocity $U$, following the numerical scheme (37). Nevertheless, the gas solver requires velocities evaluated at the interface, as well as the displacement of the interface at each time step.

From the average velocity $U$, one can obtain the interfacial longitudinal velocity $\left.u\right|_{h}$ by means of the asymptotic expansion discussed in Section 3.1. Particularly, the leading-order velocity profile (16) is used to evaluate the interfacial longitudinal velocity $\left.u\right|_{h}$ at $\mathcal{O}(1)$, namely

$$
\left(\left.u\right|_{h}\right)_{i}^{n+1}=\frac{3}{2} U_{i}^{n+1}+\frac{1}{4}\left(\tau_{i}\right)_{i}^{n},
$$

where the use of the shear stress at time $n$ is due to the explicit coupling methodology which causes a delay of the evaluation process. The effect of transferring the leading-order interfacial velocity from the film to the gas is a good estimate, given that the interface is computed at $\mathcal{O}(\varepsilon)$. Indeed, first-order velocities greatly modify the interface shape, but remain very small compared to leading-order's. For what concerns the transversal interfacial velocity, the kinematic condition (10c) yields

$$
\left(\left.v\right|_{h}\right)_{i}^{n+1}=\left(\partial_{t} h\right)_{i}^{n+1}+\left(\left.u\right|_{h}\right)_{i}^{n+1}\left(\partial_{x} h\right)_{i}^{n+1},
$$

after appropriate discretization of $\partial_{x, t} h$ with central difference and backward approximations, namely

$$
\left(\partial_{x} h\right)_{i}^{n+1}=\frac{h_{i+1}^{n+1}-h_{i-1}^{n+1}}{2 \Delta x}, \quad\left(\partial_{t} h\right)_{i}^{n+1}=\frac{h_{i}^{n+1}-h_{i}^{n}}{\Delta t} .
$$

\subsubsection{ALE method development}

In this section, we discuss the development of the ALE technique. This technique is a combination of Eulerian and Lagrangian approaches, since the nodes of the grid can move arbitrarily.

In the low-Mach computational scheme previously described, we choose that grid nodes can move along the vertical direction only. Therefore, the initial rectangular shape of the cells of the gas turn possibly into trapezoid, because left and right edges remain always parallel to each other, as sketched in Figs. 7-8. Instead, we recall that the film solver is one-dimensional because of the integral formulation. Given the position of the interface at the time step $n+1$ and in the center of each film cell, say $h_{i}^{n+1}$, one can recover the position of each corresponding node relative to the equilibrium state $h_{0}$ (equal for every cell), namely

$$
\delta_{i+1 / 2}^{n+1}=\frac{h_{i+1}^{n+1}+h_{i}^{n+1}}{2}-h_{0} .
$$

Subsequently, through the relation $d h_{i+1 / 2}^{n+1}=\delta_{i+1 / 2}^{n+1}-\delta_{i+1 / 2}^{n}$, the relative motion of the interface during time is sent to the gas to update each bottom node of the grid. Note that $d h_{i+1 / 2}^{n+1}$ is the displacement of the gas mesh for all nodes in the bottom, at time $n+1$, as sketched in Fig. 7. However, we require a mesh-update methodology which generates the new mesh at each time step. Since we want to keep the grid as regular as possible with the aim to avoid strong mesh distortions, a simple algebraic law is used to transfer the interface displacement $d h_{i+1 / 2}^{n+1}$ to all other nodes in the vertical direction, but other choices are possible.

Another relevant feature of the ALE technique is the velocity assigned to each node. In this work, since the grid moves only in the $\mathrm{y}$-direction, the longitudinal component $\hat{u}$ of the ALE velocity vanishes, namely $\hat{\mathbf{u}}=(0, \hat{v})^{T}$. The $\mathrm{y}$-velocity component $\hat{v}$ is defined for every edge, based on the displacement of the nodes at both sides of the edge. It reads

$$
\hat{v}_{e}=\frac{1}{d t}\left(d h_{N 1}+d h_{N 2}\right)
$$

where subscripts $N 1$ and $N 2$ refer to the two nodes of the edge $e$. 


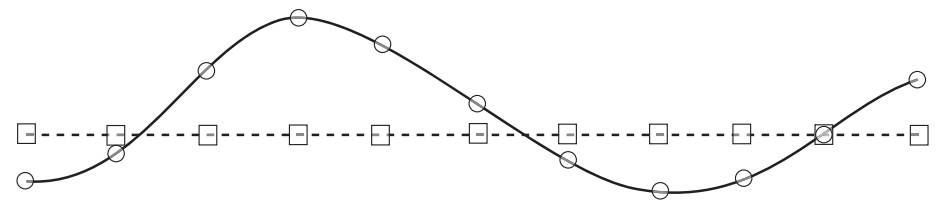

Fig. 7. Node displacements through the ALE technique at the interface liquid-gas. The dashed line indicates the uniform flow.

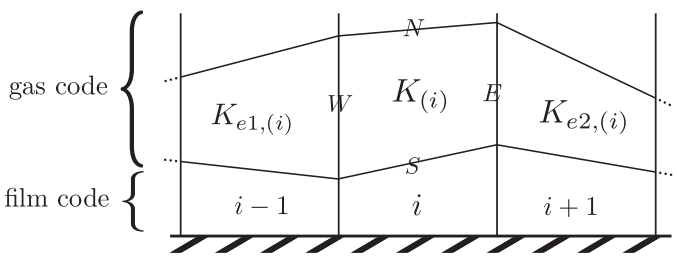

Fig. 8. Film and gas mesh: coupling between the two codes and notations.

\subsubsection{Gas to film coupling}

The last step of the coupling methodology consists in the resolution of the gas flow and in the transfer of interfacial shear stress and pressure gradient to the film. Shear stress is calculated on the bottom edges of the grid: the interfacial shear stress provided by the gas code reads

$$
\left(\tau_{i}\right)_{K}=2 \frac{\mu_{2}}{\mu_{1}}\left(\mathbf{D}_{S}^{n} \cdot \mathbf{n}_{S}^{n+1}\right) \cdot \mathbf{t}_{S}
$$

where $\mathbf{t}$ is the tangential unity vector, while the subscript $S$ refers to the South cell of the generic cell $K$, see Fig. 8.

The pressure gradient is instead given by the $\mathrm{x}$-momentum balance equation (50). Indeed, the pressure gradient in each cell is directly provided by

$$
G_{K}^{n+1}=-\frac{r}{m_{K}^{n+1}} \sum_{e \in \partial K} \Pi_{e}^{n+1} \mathbf{n}_{e, K}^{n+1} m_{e}^{n+1} .
$$

This quantity is then transfered to the film code. Precisely, the liquid film needs the pressure gradient at the interface, rather than in the center of the first cell. As a matter of fact, we obtain already a good approximation through the pressure gradient (63).

Finally, spatio-temporal derivatives of interfacial shear stress and pressure gradient are calculated as already shown in (43) and (44).

\section{Results}

In this section, we investigate the SWANS model by simulating non-linear waves occurring in two-layer co-current flows. For this purpose, two tests have been performed. The first (Section 6.1) is taken from the work by Dietze \& Ruyer-Quil [10] and consists in a strongly confined film-gas system driven by pressure gradient in a horizontal channel. Our computational analysis provides spatio-temporal evolutions of the film thickness, as well as shear stress and pressure gradient fields. These are then compared to the results provided by DNS simulations of Dietze \& Ruyer-Quil by means of OpenFOAM, as well as to their full reduced model, which consists in using second-order depth-integrated equations in both layers.

The second test (Section 6.2), instead, is a vertical two-layer system driven by gravity and flowing in a large channel, meaning that the liquid film is thin compared to the wavelength of traveling waves while the gas layer is not. Therefore, a full reduced model cannot be applied in this configuration, unlike the SWANS model presented here. This is one of the main point of our work. The spatio-temporal evolution of the film thickness is compared to DNS performed with the code Slosh of ONERA $[47,48]$.

\subsection{Horizontal two-layer flow}

This test is taken from Dietze \& Ruyer-Quil [10], which has been suggested by the work of Frank [9]. A liquid film flows in a horizontal channel sheared by a confined laminar gas. Such a two-layer flow is pressure-driven and develops in a strictly narrow channel. The uniform film thickness $h_{1}=0.13 \mathrm{~mm}$ is $1 / 3$ of the channel height.

This strongly confined flow has wide applications in coolers and distillation columns, where heat and mass transfers are relevant processes. Following Frank [9], gravity is not considered as may happen for film flowing under micro-gravity conditions. Finally, Table 1 shows the values of $2 \pi h L_{x}^{-1}$ for the liquid and the gas: the long-wave theory can be applied to both the layers, being $k h<<1$. 
Table 1

Dimensionless thickness $2 \pi h L_{x}^{-1}$ and dimensionless numbers for the horizontal confined channel test and the vertical large channel one. In this case $\hat{R} e_{2}=R e_{2} \tilde{U}_{2} \tilde{U}_{0}^{-1} \tilde{L} \tilde{h}_{0}^{-1}$ with $\tilde{U}_{2}$ average gas velocity and $\tilde{L}$ length of the channel.

\begin{tabular}{llcccrrr}
\hline Test & Channel & $2 \pi h_{1} L_{x}^{-1}\left(\cdot 10^{-2}\right)$ & $2 \pi h_{2} L_{x}^{-1}\left(\cdot 10^{-2}\right)$ & $R e_{1}$ & $\hat{R e}$ & $F r$ \\
\hline Horizontal & narrow & 3.14 & 6.28 & 4.48 & 36.8 & 0.93 \\
Vertical & large & 20.5 & 748.7 & 16.5 & 1972 & 5.002 \\
\hline
\end{tabular}

Table 2

Physical properties of the air-water flow used for the two-layer validation test.

\begin{tabular}{lllll}
\hline Fluid & $\rho\left(\mathrm{kg} \mathrm{m}^{-3}\right)$ & $v\left(10^{-5} \mathrm{~m}^{2} \mathrm{~s}^{-1}\right)$ & $\gamma\left(10^{-3} \mathrm{Nm}^{-1}\right)$ & $\rho_{2} / \rho_{1}$ \\
\hline Water & 1000 & 0.1 & 76.9 & $\mu_{2} / \mu_{1}$ \\
Air & 1 & 1 & & 0.001 \\
\hline
\end{tabular}

Table 3

Meshes used for the horizontal test, and comparison with the one from Dietze \& Ruyer-Quil's DNS. Note that, by construction, SWANS has only one vertical cell in the liquid layer.

\begin{tabular}{llllc}
\hline Mesh & $\Delta x(\mu \mathrm{m})$ & $\Delta y(\mu \mathrm{m})$ & \multicolumn{1}{c}{$\Delta x / \Delta y$} & Cell number $\left(\cdot 10^{3}\right)$ \\
\hline SWANS & 54.16 & 10.83 & 5 & 12 \\
Diet. \& RQ. (DNS) & 11.25 & 7.5 & 1.5 & 120 \\
\hline
\end{tabular}

\subsubsection{Initial flow conditions}

The initial conditions coincide with the uniform flow with a sinusoidal perturbation:

$$
\begin{aligned}
& h=h_{0}\left[1+\epsilon \sin \left(2 \pi \frac{x}{\lambda}\right)\right], \\
& q=q_{0}\left[1+\epsilon \sin \left(2 \pi \frac{x}{\lambda}\right)\right],
\end{aligned}
$$

with $\epsilon=0.25$ for comparison with Dietze \& Ruyer-Quil. A pressure jump of $\Delta p=64$ Pa is imposed between the entry and exit sides of the channel, and the velocity profiles are parabolic and match at the interface for $\left.u\right|_{h}=0.0621 \mathrm{~m} \mathrm{~s}^{-1}$, in the uniform flow. Interacting fluids are water and air, whose physical properties are listed in Table 2. Dimensionless numbers are described in Table 1, while mesh data can be found in Table 3.

\subsubsection{The SWANS model versus DNS and two-layer long-wave model}

Fig. 9 shows the time evolution of the film thickness consequence of the perturbed equilibrium (64). At the beginning, interfacial waves develop before the achievement of periodic conditions, characterized by periodic waves composed by a main hump anticipated by capillary ripples. In order to validate the SWANS model, we provide in this section comparisons with the DNS from Dietze \& Ruyer-Quil, as well as with their two-layer second-order long-wave model [10]. Fig. 10 compares the film thickness of the traveling wave normalized with the channel height $H$. Firstly, one can notice that traveling waves reach large levels of amplitude, by occupying more than half of the entire channel height, resulting in a strong reduction of the gas cross-section. Secondly, the comparison of SWANS (solid line) with the DNS (dots) and the full second-order long-wave model (dashed line) shows good agreement: the deviation at the peak level is almost $5 \%$. A larger difference between SWANS and the DNS arises in the capillary region. However, we recall that first-order long-wave models miss second-order stream-wise diffusion and this manifests itself mostly on amplitude and phase of capillary ripples (Kalliadasis et al. [35]). In addition, our result might be affected by the grid size in the gas, as discussed in the following.

Figs. 11 and 12 show the pressure gradient and the wall shear stress at the top wall of the channel. Again, we notice a general good agreement of SWANS with the DNS and the full second-order model. Nevertheless, some disparities can be observed in the peak region, and those might be mainly due to the mesh size used for SWANS, see Table 3, since we solve Navier-Stokes equations in the gas phase and at the top wall the effect of the liquid is minor otherwise in the channel. Indeed, if our mesh is good enough for the accuracy of the wave profile in Fig. 10, it needs to be refined to capture in an excellent way the stresses at the top wall. Furthermore, we recall that the discretization of the Navier-Stokes equations is first-order in space and time, and that the mesh-update methodology described in Section 5.3.2 plays on the result accuracy when the mesh is not fine enough.

Concerning the liquid, Fig. 13 shows the wall shear stress at the bottom wall. In this case, a more important discrepancy can be observed between SWANS and the DNS. The wall shear stress is computed using the definition (18) plus the correction (22). Given that the correction is very small, the definition (18) can elucidate that the flaw in this comparison is mostly due to the interfacial shear stress: Fig. 14 compares SWANS with the full second-order model of Dietze \& Ruyer-Quil, noticing a general good agreement, although some differences can be detected. As explained above, those are given to the mesh convergence, being of the same order as the top wall stresses, but might be also due to the coupling methodology, whose limitations have been already discussed in Section 5. Therefore, the difference affecting $\tau_{i}$ in Fig. 14 plays directly on the wall shear stress $\tau_{w 0}$ in Fig. 13, in addition to the omission of second-order terms in our film model. 


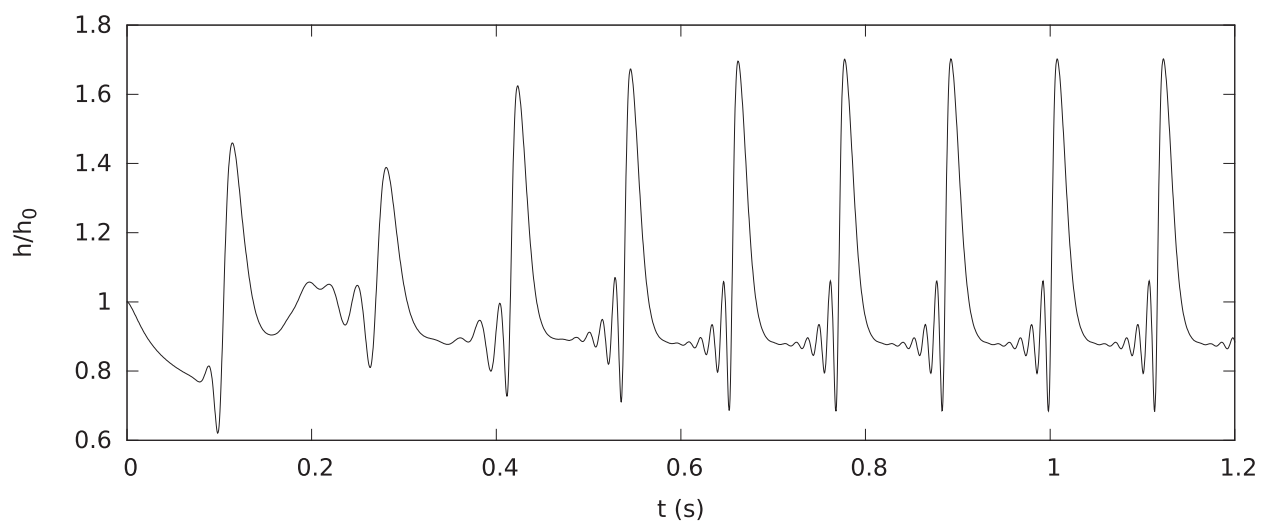

Fig. 9. Time evolution of the film thickness at $x=0$ normalized with the uniform flow. Dimensionless numbers in Table 1 (horizontal test).

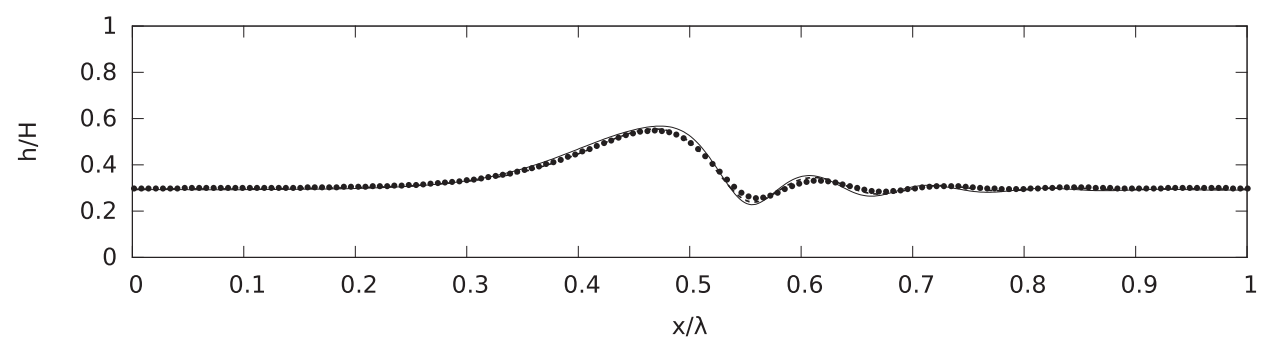

Fig. 10. Traveling wave in the channel at $t=0.75 \mathrm{~s}$. Comparison of DNS (dots) and the two-layer long-wave model (dashed line) of Dietze \& Ruyer-Quil, Fig. 7(a) of [10], with SWANS (solid line). Mesh sizes are given in Table 3, and dimensionless numbers in Table 1 (horizontal test).

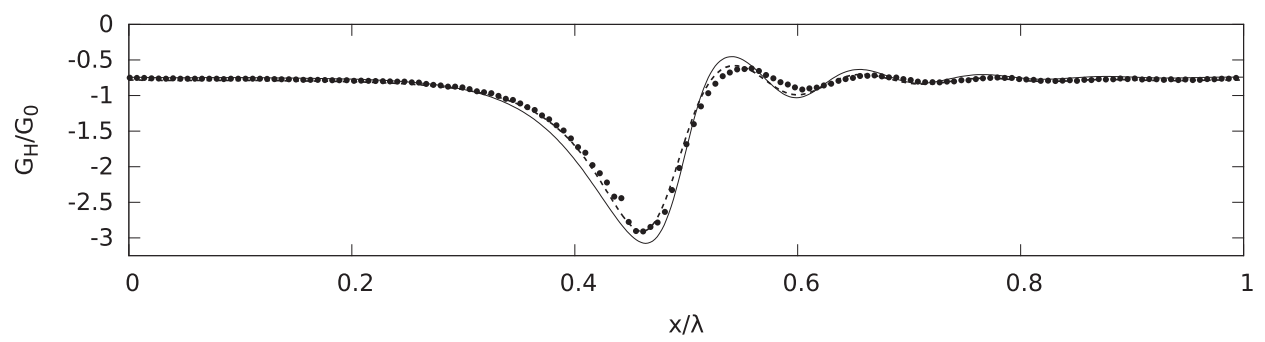

Fig. 11. Pressure gradient at the top wall at $t=0.75 \mathrm{~s}$ normalized to the uniform value $G_{0}=-\Delta p / L=2461.5$ Pa $\mathrm{m}^{-1}$. Comparison of DNS (dots) and the two-layer long-wave model (dashed line) of Dietze \& Ruyer-Quil, Fig. 7(b) of [10], with SWANS (solid line). Mesh sizes are given in Table 3, and dimensionless numbers in Table 1 (horizontal test).

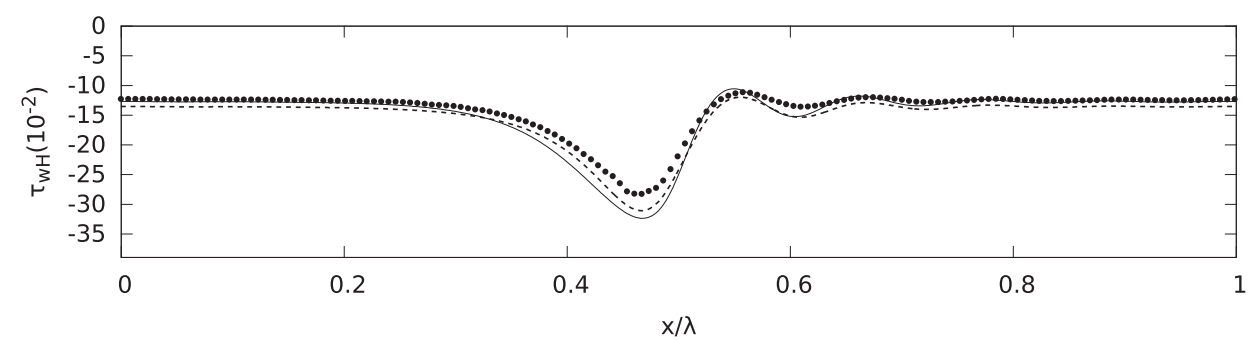

Fig. 12. Shear stress at the top wall at $t=0.75 \mathrm{~s}$ normalized with $\rho_{2} \tilde{U}_{2}^{2}=1.96 \cdot 10^{-4} \mathrm{~Pa}$. Comparison of DNS (dots) and the two-layer long-wave model (dashed line) of Dietze \& Ruyer-Quil, Fig. 7(c) of [10], with SWANS (solid line). Mesh sizes are given in Table 3, and dimensionless numbers in Table 1 (horizontal test).

As final comparison, Fig. 15 shows the interfacial pressure gradient, to which the same conclusions as the interfacial shear stress can be assumed to explain the divergence from the full second-order model.

Finally, these detailed comparisons demonstrate that our model SWANS can capture well the non-linear phenomena occurring in confined two-layer flows. 


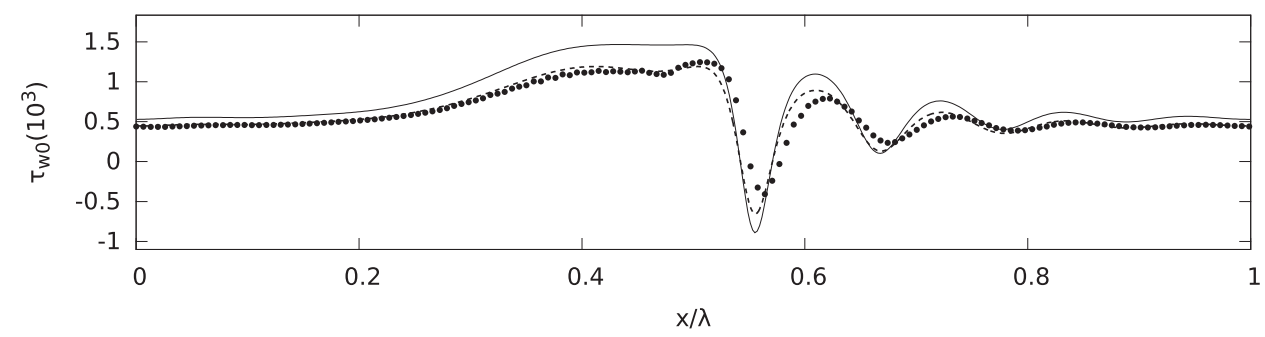

Fig. 13. Shear stress at the bottom wall at $t=0.75 \mathrm{~s}$ normalized with $\rho_{1} \tilde{U}_{0}^{2}=1.09$ Pa. Comparison of DNS (dots) and the two-layer long-wave model (dashed line) of Dietze \& Ruyer-Quil, Fig. 7(d) of [10], with SWANS (solid line). Mesh sizes are given in Table 3, and dimensionless numbers in Table 1 (horizontal test).

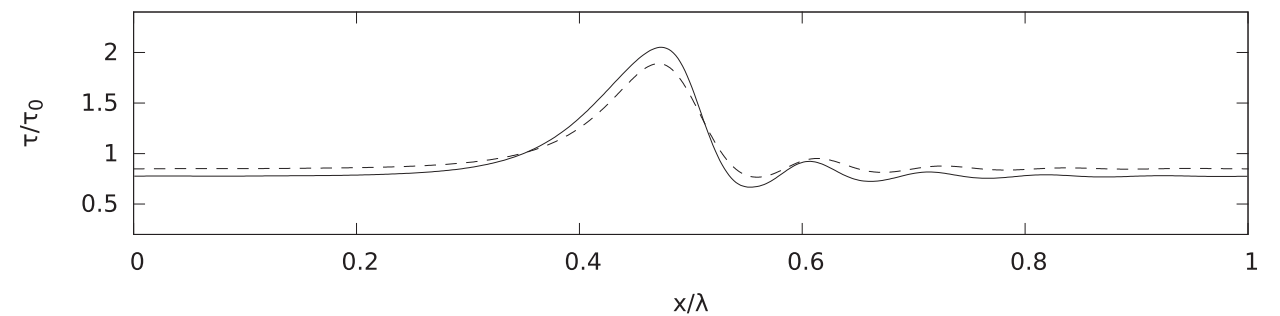

Fig. 14. Space evolution at $t=0.75 \mathrm{~s}$ of interfacial shear stress normalized to the uniform value $\tau_{i 0}=0.32$ Pa. Comparison between the two-layer long-wave model of Dietze \& Ruyer-Quil (dashed line), Fig. 8(c) of [10], and SWANS (solid line). Dimensionless numbers in Table 1 (horizontal test), and SWANS mesh in Table 3.

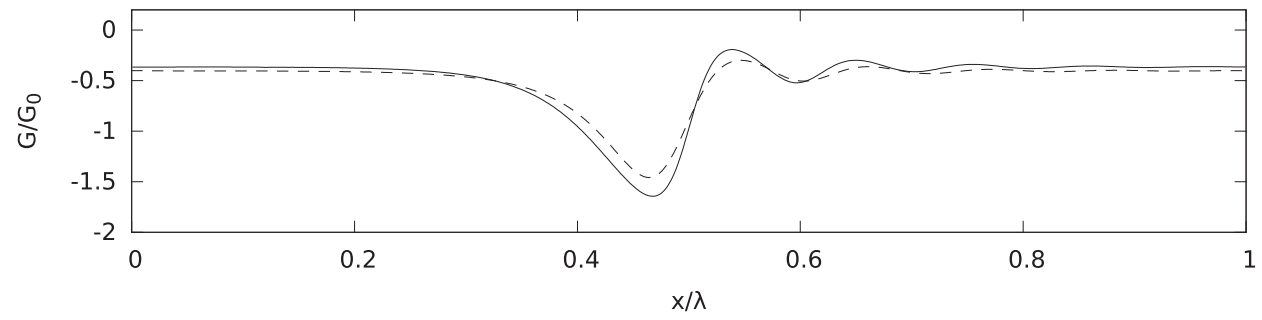

Fig. 15. Space evolution at $t=0.75 \mathrm{~s}$ of interfacial pressure gradient normalized to the uniform value $G_{0}=-\Delta p / L=2461.5$ Pa $\mathrm{m}^{-1}$. Comparison between the two-layer long-wave model of Dietze \& Ruyer-Quil (dashed line), Fig. 8(b) of [10], and SWANS (solid line). Dimensionless numbers in Table 1 (horizontal test), and SWANS mesh in Table 3.

\subsubsection{Analysis of compressibility effects}

We recall that we use compressible Navier-Stokes equations in the gas phase, and solve flows at very low speed, such as the two-layer horizontal flow described above. As a consequence, since classical solvers can be very dissipative in those regimes, a low-Mach scheme has been used to discretize the equations, which allows solving this shortcoming. However, given the use of compressible equations, some compressibility effects can arise due to the extremely confined configuration used in the horizontal test. With the aim to check whether the solution has been modified by the presence of compressibility effects, Fig. 16 shows the Mach number and the density (or pressure) distribution into the channel. We firstly note that the Mach number is very low, as expected, due to the moderate gas speed. Noteworthy is that the analysis of the density field shows piece-wise variations along the stream-wise direction of the channel. This explains that the presence of the wave, although of great amplitude, does not modify the density field in the cross-stream direction, suggesting that no compressibility effects appear.

Meanwhile, one can notice that the pressure (or the density) does not increase in the capillary region just after the main hump, although there exists a widening of the effective gas section: this shows that in addition to the inviscid contribution, i.e. Bernoulli velocity and pressure distributions, also the shear stress plays an important role in such a test.

\subsection{Vertical two-layer flow}

In the second test, we investigate a gravity-driven air-water system flowing in a vertical large channel. In such a configuration, the long-wave theory can be applied to the liquid film only, because the gas thickness is of the same order as the imposed wavelength $\lambda=5.2 \mathrm{~mm}$, see Table 1 . The uniform flow is disturbed according to $h=h_{0}[1+\epsilon \sin (2 \pi x / \lambda)]$ (and same for the flow rate $q$ ), where $\epsilon=0.25$ and $h_{0}=0.17 \mathrm{~mm}$. Dimensionless numbers are described in Table 1 . The total flow rate is almost entirely given by the gas, being $q_{2} / q_{1}=1194$ the ratio of flow rates. 

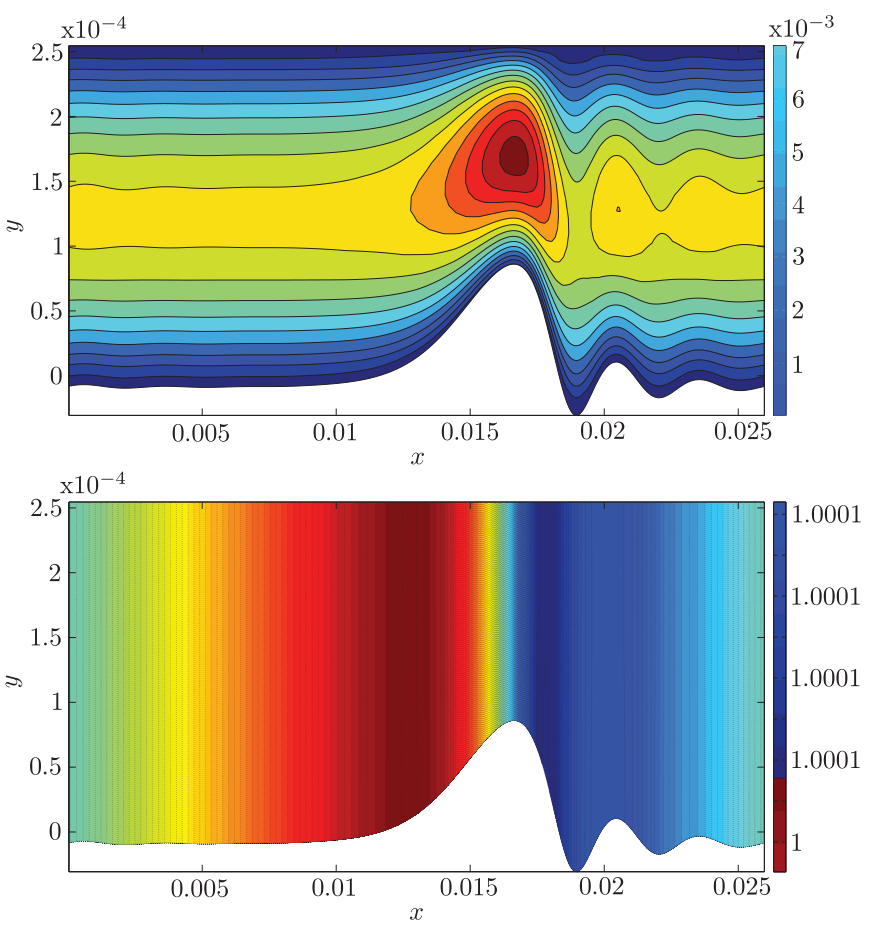

Fig. 16. (top) Mach number contour in the gas phase. (bottom) Density contour $\left(\mathrm{kg} \mathrm{m}^{-3}\right)$, or pressure contour, in the gas phase. The liquid film is white. Dimensionless numbers in Table 1 (horizontal test).

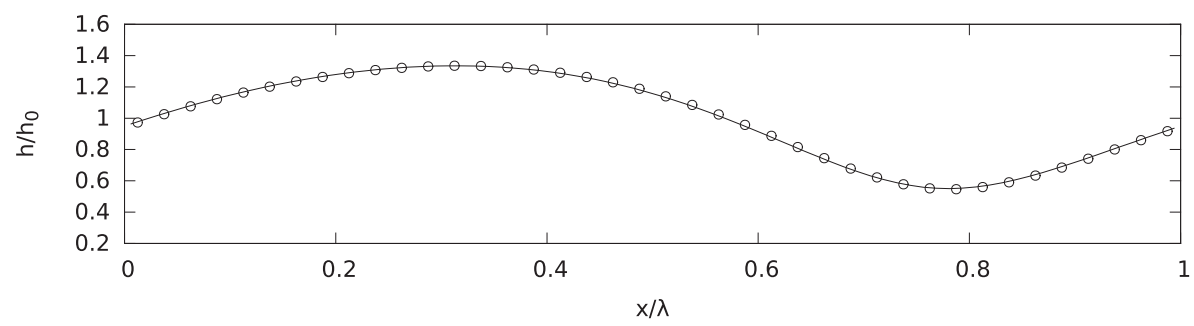

Fig. 17. Film thickness versus normalized wavelength for saturated waves at $t=0.165 \mathrm{~s}$. Convergence of two different meshes. Solid line: mesh SWANS2 in Table 4, circles: mesh SWANS1 in Table 4. Dimensionless numbers in Table 1 (vertical test).

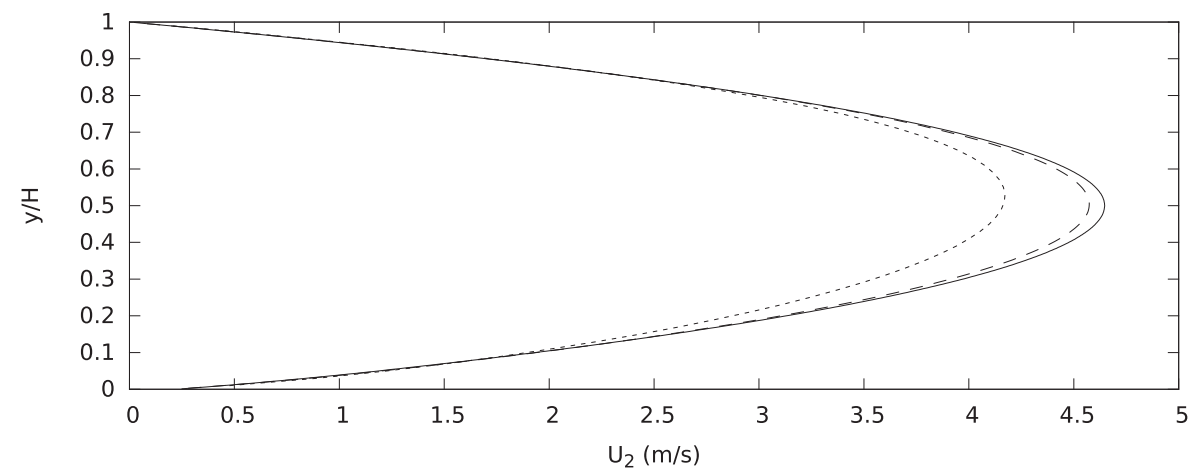

Fig. 18. Gas velocity profiles above saturated waves. Convergence of three different meshes (Table 4). Solid line: SWANS3; dashed line: SWANS2; dotted line: SWANS1. Dimensionless numbers in Table 1 (vertical test), and $H=6.37 \mathrm{~mm}$.

Fig. 17 shows the space evolution of the saturated waves for two different meshes: the convergence on the film thickness is clear. However, convergence on the gas velocity profiles is sketched in Fig. 18, according to which the coarsest mesh (mesh SWANS1 of Table 4) does not provide a proper solution, although seems to be converged on the film side (Fig. 17). 
Table 4

Meshes used in this work, from the coarsest to the finest, and those used in Slosh.

\begin{tabular}{lcccc}
\hline Mesh & $\Delta x(\mu \mathrm{m})$ & $\Delta y(\mu \mathrm{m})$ & $\Delta x / \Delta y$ & \multicolumn{1}{c}{ Cell number $\left(\cdot 10^{3}\right)$} \\
\hline SWANS1 & 130 & 124 & 2.05 & 2.1 \\
SWANS2 & 65 & 31 & 8.39 & 16 \\
SWANS3 & 130 & 15.5 & 4.2 & 16 \\
Slosh coarse (DNS) & 65 & 15.5 & 3.75 & 16 \\
Slosh fine (DNS) & 30 & 8 & 140 \\
\hline
\end{tabular}

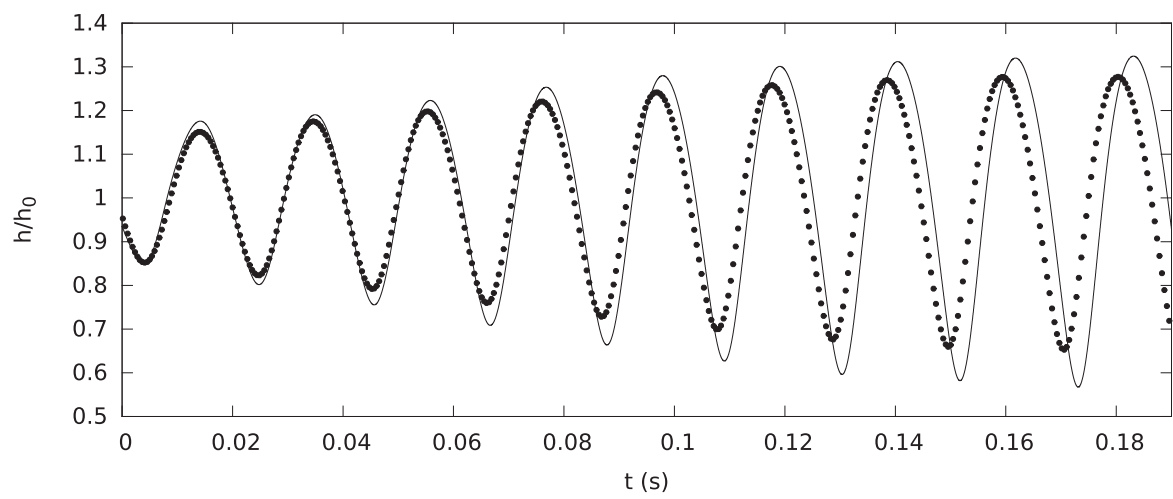

Fig. 19. Film thickness time evolution at $x=0$. Comparison between SWANS (solid line) using mesh SWANS2 of Table 4 and our DNS (dots), using the finest mesh in Table 4. Dimensionless numbers in Table 1 (vertical test), and $h_{0}=0.17 \mathrm{~mm}$.

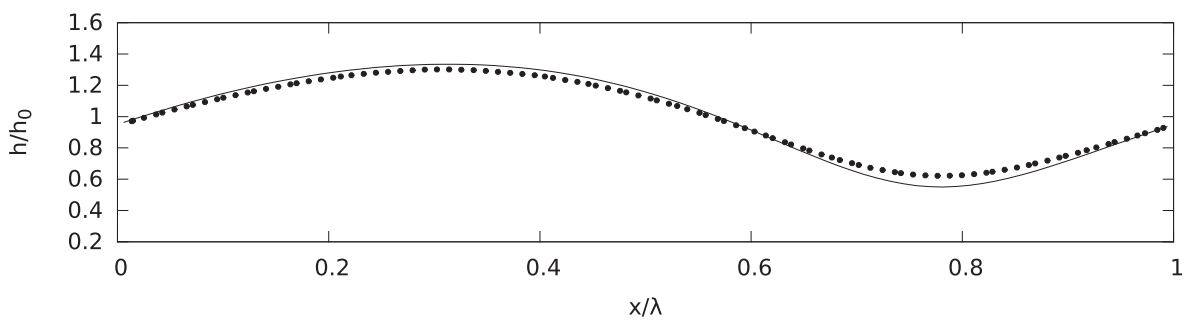

Fig. 20. Film thickness space evolution of saturated non-linear waves at $t=0.165 \mathrm{~s}$. Comparison between SWANS (solid line) using mesh SWANS2 of Table 4 and our DNS (dots), using the finest mesh in Table 4. Dimensionless numbers in Table 1 (vertical test).

\subsubsection{The SWANS model versus DNS analysis}

Film thickness spatio-temporal evolutions have been compared with our DNS simulations provided by means of the ONERA platform Slosh $[47,48]$. The Slosh solver is based on a compressible two-fluid model in which both immiscible fluids are supposed to be simultaneously present at any given point and satisfy the local mechanical equilibrium. The interface between both fluids is supposed to be diffuse, i.e. without interface reconstruction, and surface tension effects are taken into account through the Continuous Surface Stress (CSS) formulation [49]. Concerning the numerical method, this model is solved by using a conservative cell centered finite volume approach on unstructured mesh with an accurate low-Mach scheme [42]. Comparison of the time evolution of the film thickness in Fig. 19 shows good agreement. However, a small discrepancy in the wave frequency of about 5\% appears, as well as in the amplitude of the humps. Fig. 20 compares the profiles of saturated non-linear waves; we again notice a good agreement between SWANS and DNS. However, some discrepancies appearing in the amplitude can be explained by the second-order dissipation missing in our long-wave thin film model, as discussed in the next section.

Noteworthy is also that DNS requires much more cells to achieve convergence, as compared to the SWANS model (see Table 4).

\subsubsection{Effect of second-order dissipation in the film}

Finally, we study the effect of the second-order dissipative terms on the SWANS results previously described. Indeed, Ruyer-Quil \& Manneville [20] have shown, for gravity-driven liquid films, that second-order dissipative terms play an important role in the accuracy of long-wave models.

Including $\mathcal{O}\left(\varepsilon^{2}\right)$ terms, the $\mathrm{x}$-momentum equation (12) becomes

$$
\partial_{t} u+u \partial_{x} u+v \partial_{y} u=-\partial_{x} p+\frac{\sin \beta}{F r}+\frac{1}{R e}\left(\partial_{y y} u+\partial_{x x} u\right)+\frac{1}{W e} \partial_{3 x} h,
$$




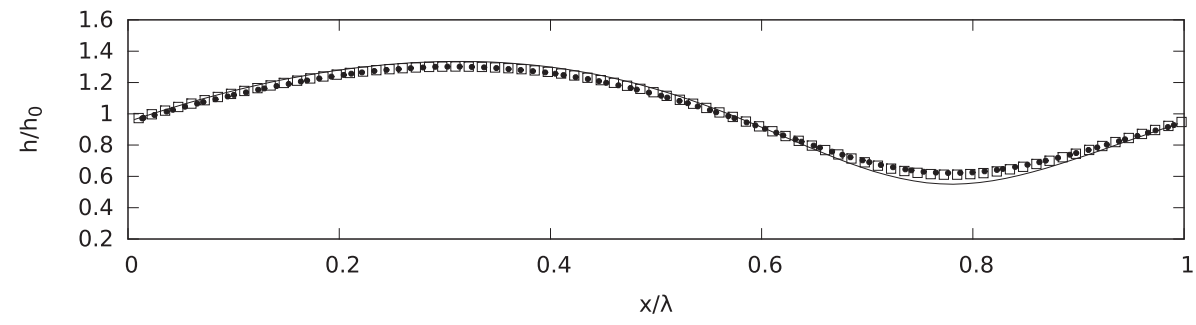

Fig. 21. Film thickness space evolution of the traveling wave at $t=0.165 \mathrm{~s}$. Comparison between SWANS by adding the dissipative term $2 \partial_{x x} q$ (squares), using the mesh SWANS2 of Table 4, and our DNS (dots) with the finest mesh of Table 4. Solid line is solution by SWANS without second-order dissipation with mesh SWANS2 of Table 4. Dimensionless numbers in Table 1 (vertical test).

with

$$
\partial_{x} p=-G+\frac{\cos \beta}{F r} \partial_{x} h-\frac{1}{W e} \partial_{3 x} h+\frac{1}{R e} \partial_{x}\left(\left.\partial_{y} v\right|_{h}+\partial_{y} v\right)-\frac{2}{R e}\left[\left.\partial_{x x} h \partial_{y} u\right|_{h}+\partial_{x} h \partial_{x}\left(\left.\partial_{y} u\right|_{h}\right)\right] .
$$

The analysis of all second-order terms goes beyond this work, and we focus only on the second-order dissipative term $2 \partial_{x x} u$ (note that the coefficient 2 comes from the equivalence $\partial_{x y} v=-\partial_{x x} u$ and $\left.\partial_{y} v\right|_{h}=-\left.\partial_{x} u\right|_{h}$ arising from the continuity equation). The integration of such a term over the film thickness provides $2 \partial_{x x} q$. Fig. 21 shows that the simple addition of the dissipative term $2 \partial_{x x} q$ improves the comparison with DNS.

\section{Conclusions}

We have investigated the two-dimensional non-linear dynamics of thin liquid films sheared by a laminar gas flow in horizontal or inclined channels. So far, this problem has been studied either with DNS, i.e. Navier-Stokes equations applied to both the phases (Frank [8,9], among the others), or with a full low-dimensional model, based on depth-integrated equations applied to the film and the gas (Dietze \& Ruyer-Quil [10]). The former manifests a too great computational cost when studying industrial applications. The latter can be instead applied only to extremely narrow channels, where the thickness of the gas flow remains very thin compared to the wavelength of interfacial waves.

In this paper, in order to get rid of the above mentioned limitations, a coupling methodology between depth-integrated equations and compressible Navier-Stokes equations, for the liquid and gas phases respectively, has been provided.

By focusing on the liquid problem first, a system of consistent depth-integrated equations accurate at order one in the film parameter $\varepsilon<<1$ has been developed. This long-wave model captures exactly the linear stability threshold of long interfacial waves for thin liquid films driven by shear stress and pressure gradient, and possibly by the gravity (a detailed linear stability analysis of this long-wave model for gravity-driven liquid films sheared by a constant stress can be found in Lavalle et al. [36]); furthermore, it is able to capture the long-wave instabilities in the non-linear regime.

Extending the work of Noble \& Vila [39] to sheared films, these depth-integrated equations have been discretized by means of an augmented system, which accounts for an evolution equation for the surface tension. This avoids numerical instability while using classical centered approximations.

As for the gas flow, compressible Navier-Stokes equations have been used to describe interactions with the wavy liquid film. In order to study flows at low speed, while avoiding dissipation of classical approximate Riemann solvers, we have used a low-Mach scheme to discretize the compressible Navier-Stokes equations, in line with the work of Grenier et al. [42] (for two-fluid flows). In addition, we have accounted for moving meshes in the gas domain, by implementing the ALE technique. Particularly, this allows moving the nodes of the gas grid following the position of the liquid-gas interface.

The numerical code SWANS predicts well the evolution of the liquid-gas interface in space and time. Indeed, two numerical tests have been performed: confined channels, where the long-wave theory is still valid for the gas layer, and large channels, where the long-wave approximation can be applied to the liquid phase only. In the first test, profiles of developed non-linear waves and stress fields have been compared with good agreement to DNS by Dietze \& Ruyer-Quil [10]. Furthermore, shear stress and pressure gradient have been compared with agreement to those obtained by means of the above-mentioned DNS and the full integral model (simplified second-order model of Dietze \& Ruyer-Quil). The mismatch can be explained in a first stage with the mesh accuracy and the omission of second-order dissipation in the liquid model. We have also confirmed that any compressibility effects changes the solution, although we adopt compressible Navier-Stokes equations for flows at very low speed.

In the second performed test, spatio-temporal evolutions of the liquid-gas interface have been compared with good agreement to DNS by means of the platform Slosh $[47,48]$. We have also confirmed that such comparisons improve when adding second-order terms in our film model (Kalliadasis et al. [35]).

In comparison with existing models and DNS technique, our methodology based on the coupling between depthintegrated equations and Navier-Stokes equations has the following main advantages: $(i)$ it captures the long-wave linear stability threshold and non-linear instabilities for sheared liquid films, thanks to the first-order long-wave model; (ii) it 
extends the full two-layer low-dimensional model to large channels, and thus allows considering a wide number of configurations and industrial applications; (iii) it allows adopting greater time step in the Navier-Stokes analysis thanks to the presence of the ALE technique, which transfers the interface position from the film to the gas and keeping the informations on the surface tension and hydrostatic pressure field; (iv) it avoids numerical instability of the dispersive term in the film model thanks to the augmented system technique, which produces an evolution equation for the surface tension, while decreasing the order of the differential system of equations.

For what concerns the extension of this work, accounting for dissipative second-order terms would allow understanding the limit of first-order film models in the comparison of non-linear waves with the DNS. Secondly, one could develop an implicit scheme for surface tension, in order to save further computational cost with respect to DNS. Finally, the most interesting task would be to extend SWANS to boundary-layer flows, either laminar or turbulent, over wavy liquid films. Heat and mass transfers between the two phases might also be considered.

\section{Acknowledgements}

The authors acknowledge G. Dietze for having provided DNS and full model data from [10], and C. Ruyer-Quil and S. Popinet for stimulating remarks. Also, they thank one of the anonymous referee for encouraging further comparisons, i.e. Figs. 11-13.

\section{References}

[1] W.H. Henstock, T.J. Hanratty, Gas absorption by a liquid layer flowing on the wall of a pipe, AIChE J. 25 (1) (1979) 122-131.

[2] M.J. McCready, T.J. Hanratty, Effect of air shear on gas absorption by a liquid film, AIChE J. 31 (1985) 2066.

[3] T.R. Salamon, R.C. Armstrong, R.a. Brown, Traveling waves on vertical films: numerical analysis using the finite element method, Phys. Fluids 6 (6) (1994) 2202-2220, http://dx.doi.org/10.1063/1.868222.

[4] Y.Y. Trifonov, Stability and bifurcations of the wavy film flow down a vertical plate: the results of integral approaches and full-scale computations, Fluid Dyn. Res. 44 (3) (2012), http://dx.doi.org/10.1088/0169-5983/44/3/031418.

[5] B. Ramaswamy, S. Chippada, S.W. Joo, A full-scale numerical study of interfacial instabilities in thin-film flows, J. Fluid Mech. 325 (2006) 163-194, http://dx.doi.org/10.1017/S0022112096008075.

[6] J. Li, Y.Y. Renardy, M. Renardy, A numerical study of periodic disturbances on two-layer Couette flow, Phys. Fluids 10 (12) (1998) 3056-3071, http:// dx.doi.org/10.1063/1.869834.

[7] J. Zhang, M.J. Miksis, S.G. Bankoff, G. Tryggvason, Nonlinear dynamics of an interface in an inclined channel, Phys. Fluids 14 (6) (2002) 1877-1885, http://dx.doi.org/10.1063/1.1475313.

[8] A.M. Frank, Shear driven solitary waves on a liquid film, Phys. Rev. E 74 (6) (2006) 065301, http://dx.doi.org/10.1103/PhysRevE.74.065301.

[9] A.M. Frank, Numerical simulation of gas driven waves in a liquid film, Phys. Fluids 20 (12) (2008) 122102, http://dx.doi.org/10.1063/1.3053827.

[10] G.F. Dietze, C. Ruyer-Quil, Wavy liquid films in interaction with a confined laminar gas flow, J. Fluid Mech. 722 (2013) 348-393, http://dx.doi.org/ $10.1017 / \mathrm{jfm} .2013 .98$.

[11] S. Popinet, An accurate adaptive solver for surface-tension-driven interfacial flows, J. Comput. Phys. 228 (16) (2009) 5838-5866, http://dx.doi.org/ 10.1016/j.jcp.2009.04.042.

[12] Y.Y. Trifonov, Counter-current gas-liquid wavy film flow between the vertical plates analyzed using the Navier-Stokes equations, AIChE J. 56 (8) (2010) 1975-1987.

[13] P.L. Kapitza, Wave flow of a thin viscous fluid layers, Zh. Eksp. Teor. Fiz. 18 (1) (1948)

[14] J. Liu, J.P. Gollub, Solitary wave dynamics of film flows, Phys. Fluids 6 (5) (1994) 1702-1712.

[15] S.V. Alekseenko, V.Y. Nakoryakov, B.G. Pokusaev, Wave formation on a vertical falling liquid film, AIChE J. 31 (9) (1985) $1446-1460$.

[16] A.D.D. Craik, Wind-generated waves in thin liquid films, J. Fluid Mech. 26 (02) (1966) 369-392, http://dx.doi.org/10.1017/S0022112066001289.

[17] L.S. Cohen, T.J. Hanratty, Generation of waves in the concurrent flow of air and a liquid, AIChE J. 11 (1) (1965) $138-144$.

[18] D.J. Benney, Long waves on liquid films, J. Math. Phys. 45 (1966) 150-155.

[19] V.Y. Shkadov, Wave flow regimes of a thin layer of viscous fluid subject to gravity, Izv. Akad. Nauk SSSR, Meh. Židk. Gaza 2 (1) (1967) 43-51.

[20] C. Ruyer-Quil, P. Manneville, Modeling film flows down inclined planes, Eur. Phys. J. B 6 (2) (1998) 277-292, http://dx.doi.org/10.1007/s100510050550.

[21] C. Ruyer-Quil, P. Manneville, Improved modeling of flows down inclined planes, Eur. Phys. J. B 15 (2000) 357-369.

[22] B.S. Tilley, S.H. Davis, S.G. Bankoff, Nonlinear long-wave stability of superposed fluids in an inclined channel, J. Fluid Mech. 277 (1994) $55-83$.

[23] O.K. Matar, C.J. Lawrence, G.M. Sisoev, Interfacial dynamics in pressure-driven two-layer laminar channel flow with high viscosity ratios, Phys. Rev. E 75 (5) (2007), http://dx.doi.org/10.1103/PhysRevE.75.056314.

[24] G.M. Sisoev, O.K. Matar, D. Sileri, C.J. Lawrence, Wave regimes in two-layer microchannel flow, Chem. Eng. Sci. 64 (13) (2009) 3094-3102, http:// dx.doi.org/10.1016/j.ces.2009.03.044.

[25] L.A. Jurman, M.J. McCready, Study of waves on thin liquid films sheared by turbulent gas flows, Phys. Fluids A, Fluid Dyn. 1 (3) (1989) 522-536, http://dx.doi.org/10.1063/1.857553.

[26] D. Tseluiko, S. Kalliadasis, Nonlinear waves in counter-current gas-liquid film flow, J. Fluid Mech. 673 (2011) 19-59.

[27] Rajagopal Vellingiri, Dmitri Tseluiko, Nikos Savva, Serafim Kalliadasis, Dynamics of a liquid film sheared by a co-flowing turbulent gas, Int. J. Multiph. Flow 56 (2013) 93-104, http://dx.doi.org/10.1016/j.ijmultiphaseflow.2013.05.011, http://linkinghub.elsevier.com/retrieve/pii/S0301932213000840.

[28] A. Refloch, B. Courbet, A. Murrone, P. Villedieu, C. Laurent, P. Gilbank, J. Troyes, L. Tessé, G. Chaineray, J.B. Dargaud, F. Vuillot, CEDRE software, Aerosp. Lab. 2 (2011) 1-10.

[29] C.W. Hirt, A.A. Amsden, J.L. Cook, An arbitrary Lagrangian-Eulerian computing method for all flow speeds, J. Comput. Phys. 14 (1974) $227-253$.

[30] R.K.-C. Chan, A generalized arbitrary Lagrangian-Eulerian method for incompressible flows with sharp interfaces, J. Comput. Phys. 17 (1975) $311-331$.

[31] W.E. Pracht, Calculating three-dimensional fluid flows at all speeds with an Eulerian-Lagrangian computing mesh, J. Comput. Phys. 17 (1975) 132-159.

[32] A. Soulaïmani, M. Fortin, G. Dhatt, Y. Ouellet, Finite element simulation of two- and three-dimensional free surface flows, Comput. Methods Appl. Mech. Eng. 86 (1991) 265-296.

[33] P. Luchini, F. Charru, Consistent section-averaged equations of quasi-one-dimensional laminar flow, J. Fluid Mech. 656 (2010) $337-341$.

[34] P. Luchini, F. Charru, The phase lead of shear stress in shallow-water flow over a perturbed bottom, J. Fluid Mech. 665 (2010) 516-539, http://dx.doi.org/10.1017/S0022112010004313.

[35] S. Kalliadasis, C. Ruyer-Quil, B. Scheid, M.G. Velarde, Falling Liquid Films, Springer, 2012. 
[36] G. Lavalle, C. Laurent, F. Charru, J.-P. Vila, Study of the linear stability analysis of thin liquid films, in: 8th International Conference on Multiphase Flow ICMF 2013, Jeju, Korea, 2013.

[37] G. Lavalle, Integral modeling of liquid films sheared by a gas flow, Ph.D. thesis, ISAE, December 2014.

[38] C. Habchi, H. Foucart, T. Baritaud, Influence of the wall temperature on the mixture preparation in DI gasoline engines, Oil Gas Sci. Technol. 54 (1999) $211-222$.

[39] P. Noble, J.-P. Vila, Stability theory for difference approximations of some dispersive shallow water equations and application to thin film flows, SIAM J. Numer. Anal. 52 (6) (2014) 2770-2791, http://dx.doi.org/10.1137/130918009.

[40] E.F. Toro, Riemann Solvers and Numerical Methods for Fluid Dynamics, A Practical Introduction, Springer, 1997.

[41] G.B. Whitham, Linear and Nonlinear Waves, Wiley-Interscience, 1974.

[42] N. Grenier, J.-P. Vila, P. Villedieu, An accurate low-Mach scheme for a compressible two-fluid model applied to free-surface flows, J. Comput. Phys. 252 (2013) 1-19, http://dx.doi.org/10.1016/j.jcp.2013.06.008.

[43] Y. Coudière, J.-P. Vila, P. Villedieu, Convergence rate of a finite volume scheme for a two dimensional convection-diffusion problem, Math. Model. Numer. Anal. 33 (3) (1999) 493-516.

[44] C. Farhat, P. Geuzaine, C. Grandmont, The discrete geometric conservation law and the nonlinear stability of ALE schemes for the solution of flow problems on moving grids, J. Comput. Phys. 174 (2001) 669-694, http://dx.doi.org/10.1006/jcph.2001.6932.

[45] J. Donea, A. Huerta, J.-P. Ponthot, A. Rodriguez-Ferran, Arbitrary Lagrangian-Eulerian Methods, 2004.

[46] N. Lanson, J.-P. Vila, Renormalized meshfree schemes I: consistency, stability, and hybrid methods for conservation laws, SIAM J. Numer. Anal. 46 (4) (2008).

[47] G. Chanteperdrix, P. Villedieu, J.-P. Vila, A compressible model for separated two-phase flows computations, in: ASME 2002 Joint U.S.-European Fluids Engineering Division Conference, 2002.

[48] N. Schied, P. Villedieu, J.-P. Vila, A diffuse interface model for the numerical simulation of thermocapillary effects, in: ASME 2009 Fluids Engineering Division Summer Meeting, 2009.

[49] D. Gueyffier, J. Li, A. Nadim, R. Scardovelli, S. Zaleski, Volume-of-fluid interface tracking with smoothed surface stress methods for three-dimensional flows, J. Comput. Phys. 152 (1999) 423-456. 